\title{
Riccati Observers for the Non-stationary PnP Problem
}

\author{
Tarek Hamel and Claude Samson
}

\begin{abstract}
This paper revisits the problem of estimating the pose (position and orientation) of a body in 3D space with respect to (w.r.t.) an inertial frame by using i) the knowledge of source points positions in the inertial frame, ii) the measurements of the body angular velocity expressed in the body's frame iii) the measurements of the body translational velocity, either in the body frame or in the inertial frame, and iv) source points bearing measurements performed in the body frame. An important difference with the much studied static Perspective$\mathrm{n}$-Point (PnP) problem addressed with iterative algorithms is that body motion is not only allowed but also used as a source of information that improves the estimation possibilities. With respect to the probabilistic framework commonly used in other studies that develop Extended Kalman filter (EKF) solutions, the deterministic approach here adopted is better suited to point out the observability conditions, that involve the number and disposition of the source points in combination with body motion characteristics, under which the proposed observers ensure robust estimation of the body pose. These observers are here named Riccati observers because of the instrumental role played by the Continuous Riccati equation (CRE) in the design of the observers and in the Lyapunov stability and convergence analysis that we develop independently of the well-known complementary (either deterministic or probabilistic) optimality properties associated with Kalman filtering. The set of these observers also encompasses Extended Kalman filter solutions. Another contribution of the present study is to show the importance of using body motion to improve the observers performance and, when this is possible, of measuring the body translational velocity in the inertial frame rather than in the body frame to allow for the body pose estimation from a single source point taken as the origin of the inertial frame. This latter possibility finds a natural extension in the Simultaneous Localization and Mapping (SLAM) problem in Robotics.
\end{abstract}

Index Terms-Observers for nonlinear systems, observability, Perspective-n-Point problem, Riccati equation

\section{INTRODUCTION}

Body pose estimation from source points bearing measurements is well exemplified by the problem of estimating the pose of a monocular perspective camera from projected positions of observed source points measured in the camera images. When the attitude (orientation) of the camera is measured or estimated by other means, the problem reduces to the one of estimating the position of the camera. By contrast with the complete pose estimation problem, this simpler subproblem can be exactly linearised and yield observers that are globally exponentially stable under adequate observability conditions, see for instance [1]. Attitude estimation introduces

T. Hamel is with Université Côte d'Azur, CNRS, I3S, France, thamelei3s.unice.fr.

C. Samson (corresponding author) is with Université Côte d'Azur, INRIA, CNRS, I3S, France, claude.samsoneinria.fr, csamsonei3s.unice.fr. another level of complexity in relation to the structure of the compact Lie group of rotations $S O(3)$ involved in the larger group $S E(3)$ of 3D rigid transformations. In particular exact linearisation of motion equations on these groups is not possible and, even more annoying, globally convex penalty functions needed to the existence of gradient-based observers achieving global asymptotic stability of zero estimation errors do not exist. The design methodology here adopted relies on approximate linearisation in the spirit of Extended Kalman filtering (EKF), except that it is derived in a deterministic framework that allows for a clear exposition of observability conditions under which the proposed iterative observers are endowed with robust stability and convergence properties. For this reason, and also because of the instrumental role played by the Continuous Riccati Equation (CRE) in both the observers equations and the definition of the associated Lyapunov cost functions used for stability and convergence analysis, we propose the generic name of Riccati observers to refer to the class of observers here studied and whose expressions encompass EKF solutions. This point of view does not hinder a complementary probabilistic modelling of measurement perturbations that may be useful to efficiently tune the observers parameters by application of Kalman filtering rules.

Following the excellent survey [2], the perspective pose estimation problem with three source points (i.e. the minimal number needed to solve the problem in the static case when the camera and the source points are motionless) was first solved via algebraic calculations (the direct solution method) by a German mathematician in 1841 [3]. His solution consists in first determining the distances from the optic centre to the source points so as to obtain an estimation of the optic centre position expressed in the camera frame and, in a second stage, in determining the camera attitude. It was subsequently, and until recently, refined by photogrammetrists and computer vision specialists [4], [5], with complementary analyses concerning the number of poses satisfying the perspective projection equations associated with the problem and the numerical stability of the proposed solutions (study of singular solutions). This latter problem points out in particular the existence of the so-called danger cylinder and the numerical instability of the solutions when the camera optic centre is located on this cylinder (whose equation is simply obtained by zeroing the determinant of the Jacobian matrix associated with the equations [2], [6]). The extension of these analyses to four and five source points has also given rise to several publications [4], [7].

Iterative algorithms based on gradient search [8], [9] are often motivated by the observation of more than four source 
points with bearing measurement errors and possible outliers (resulting from incorrect point correspondences), with the aim of determining a pose estimate that minimizes a quadratic average error index, itself derived from the perspective projection equations. These methods are local by nature and are not directly concerned with the number of solutions to the perspective projection equations, provided that this number is finite to ensure that the error index function admits isolated (local) minima. However, the domain of convergence to a "good" solution, as well as the rate of convergence to such a solution, are related to the same regularity conditions as those associated with the stability of algebraic resolution methods. In particular, the convexity of the index function in the vicinity of the body position, which is needed to ensure robust convergence of any gradient-based algorithm to this point, requires that the rank of the Jacobian matrix associated with the perspective projection equations is equal to three. With three source points, this condition is thus again not satisfied when the optic centre is located on the danger cylinder. The analysis developed in the present paper also accounts for this type of problem via a different approach.

The approach taken in this paper may be viewed as a prolongation of the iterative approach to the more general and comparatively little studied non-stationary case involving possible body motion and on-line body pose estimation from body velocities and bearing measurements acquired over time. Related works in this direction, sharing features with the observer design methodology proposed herein, can be found in [10], [11] and references therein. Measuring body velocities is useful for many robotic applications involving state estimation and control. For this reason most robotic devices and/or their environment are equipped with sensors providing velocity information: on-board inertial measurement unit (IMU), global positioning system (GPS), proprioceptive tachometers, etc. While angular velocity is typically measured in the bodyfixed frame with gyrometers, translational body velocity can be measured either in the body-fixed frame (via tachometers for mobile robots or pitotubes for aerial vehicles) or in an inertial frame (by using a GPS or a radar mounted in the inertial frame, for instance). The fact that these two possibilities are not equivalent in terms of pose observability justifies the comparative study carried out in the paper.

We use the Automatic Control notion of uniform observability [12] to characterize the aforementioned regularity conditions and show that the explicit use of measured velocity in complementation to bearing measurements can weaken the conditions under which effective and robust pose estimation can be achieved. The relation between uniform observability and well-posedness of the Riccati equation involved in Kalman filtering is also recalled. In this respect and in the same way as the existence of solutions to the perspective projection equations does not systematically imply that the solution is numerically stable, weak observability is not sufficient to derive fast converging and robust observers. This explains the accent put in the paper on the stronger property of uniform observability.

The paper is organized as follows. Notation used throughout the paper and recalls of basic definitions related to the CRE and conditions under which a matrix-solution to a CRE associated with a time-varying linear system and its inverse are uniformly bounded are given in Section II. In Section III a generic dynamic system verifying a set of structural properties, complemented with a pre-observer system involving a CRE, is defined prior to stating conditions, reported in a theorem, under which local exponential stability of zero estimation errors is achieved for this dual system. This theorem directly applies to a number of systems evolving on $S E(3)$ with associated Riccati (EKF-like) state observers. Its application to the estimation of a body pose from velocity and source points bearing measurements is detailed in Section IV by considering two minimal parametrizations of $S E(3)$. By virtue of the theorem stated in the previous section, the Riccati observers so obtained share, despite their differences, the same local stability and convergence properties. Observers are first derived in the case where the body translational velocity is measured in the body frame (Subsection IV-B), then in the case where this velocity is measured in the inertial frame w.r.t. which the source points positions are known (Subsection IV-C). Nonuniform observability -translated in terms of source points numbers, singular geometric localizations, and body motionthat jeopardizes the performance of the observers is analysed in details in both cases. This analysis provides an alternate means to recover static singular configurations well-known in photogrammetry such as the danger cylinder, in the case of three source points, and horopter curves, in the case of four and more source points. It also points out how body motion can be useful to overcome these singularities and, perhaps more importantly, it shows that the measurement of the body translational velocity in the inertial frame combined with bearing measurement of a single source point is generically sufficient to estimate the body pose. Simulations illustrating some aspects of this analysis are reported in Section V. A short comparative analysis of stereo vs. monocular vision in terms of observability is carried out in Section VI and concluding remarks, further pointing out the connection between the single source point case and the Simultaneous Localization and Mapping (SLAM) problem in Robotics, are offered in Section VII. Finally, an extension of the approach to the case when the translational and angular body velocities are corrupted by constant additive biases, or when these velocities are constant but not measured directly, is sketched out in the Appendix.

\section{NOTATION AND DEFINITIONS}

- $|x|$ is the Euclidean norm of the vector $x \in \mathbb{R}^{n}$, and $|A|$ with $A$ a real matrix denotes the usual corresponding matrix norm.

- $x^{\top}$ is the transpose of the vector $x$, and $A^{\top}$ is the transpose of the matrix $A$.

- $\mathcal{B}_{r}^{n}=\left\{x \in \mathbb{R}^{n}:|x| \leq r\right\}$ is the closed ball in $\mathbb{R}^{n}$ of radius $r$.

- $S p^{n}=\left\{x \in \mathbb{R}^{n+1}:|x|=1\right\}$ is the $n$-dimensional sphere of radius equal to one.

- $\mathcal{S}_{+}^{n}$ is the set of symmetric non-negative semidefinite matrices of dimension $(n \times n)$.

- $0_{m \times n}$ is the null matrix with $m$ lines and $n$ columns. 
- $0_{n}$ and $I_{n}$ are respectively the null matrix and the identity matrix of dimension $n \times n$.

- $S(x)$ is the skew-symmetric matrix associated with the cross-product in $\mathbb{R}^{3}$, i.e. $S(x) y=x \times y, \forall x \in \mathbb{R}^{3}, \forall y \in$ $\mathbb{R}^{3}$

- $\Pi_{x}:=I_{3}-x x^{\top}$ with $x \in S p^{2}$ is the orthogonal projection operator in $\mathbb{R}^{3}$ onto the two-dimensional vector subspace orthogonal to $x$.

- With $f$ denoting a vector-valued function depending on the two variables $x$ and $y$, and on the time variable $t$, we write $f=O\left(|x|^{k_{1}}|y|^{k_{2}}\right)$ with $k_{1} \geq 0$ and $k_{2} \geq 0$ if $\forall t$ : $|f(x, y, t)| /\left(|x|^{k_{1}}|y|^{k_{2}}\right) \leq \gamma<\infty$ in the neighbourhood of $(x=0, y=0)$. If $f$ depends only on $x$ and $t$ then we write $f(x, t)=O\left(|x|^{k}\right)$ if $\forall t:|f(x, t)| /|x|^{k} \leq \gamma<\infty$ in the neighbourhood of $x=0$.

Given continuous $n \times n$-dimensional matrix-valued functions $A(t)$ and $V(t)$, with $V(t)$ non-negative semidefinite for all $t \in \mathbb{R}^{+}$, the controllability Grammian associated with the pair $(A, V)$ is the non-negative semidefinite matrix-valued function defined by

$$
W_{V}^{A}(t, t+\delta):=\frac{1}{\delta} \int_{t}^{t+\delta} \Phi(t, s) V(s) \Phi^{\top}(t, s) d s
$$

with $\Phi(t, s)$ the transition matrix associated with $A(t)$, i.e. such that $\frac{d}{d t} \Phi(t, s)=A(t) \Phi(t, s)$ with $\Phi(t, t)=I_{n}$.

Given a continuous $n \times n$-dimensional matrix-valued function $A(t)$, a continuous $m \times n$-dimensional matrix-valued function $C(t)$, and a continuous $m \times m$-dimensional matrixvalued function $Q(t)$, with $Q(t)$ non-negative semidefinite for all $t \in \mathbb{R}^{+}$, the Riccati observability Grammian associated with the triplet $(A, C, Q)$ is the non-negative semidefinite matrix-valued function defined by

$W_{Q}^{A, C}(t, t+\delta):=\frac{1}{\delta} \int_{t}^{t+\delta} \Phi^{\top}(s, t) C^{\top}(s) Q(s) C(s) \Phi(s, t) d s$

If $A(t)$ and $C(t)$ are bounded and if there exists $\delta>0$ and $\epsilon>0$ such that $W_{I_{n}}^{A, C}(t, t+\delta)>\epsilon I_{n}$ for all $t \geq 0$, then we say that the pair $(A, C)$ is uniformly observable. This is a short way of stating that the state of the Linear Time Varying (LTV) system

$$
\begin{aligned}
& \dot{x}=A(t) x+B(t) u \\
& y=C(t) x
\end{aligned}
$$

characterized by the pair $(A, C)$ is uniformly observable. This property implies in particular that $x(t)$ can be calculated from the knowledge of $u($.$) and y($.$) on the time interval [t, t+\delta]$ (see [12]).

Given $A(t), C(t), Q(t)$ and $V(t)$ as specified previously, the Continuous Riccati Equation (CRE) associated with the set $(A, C, Q, V)$ is

$$
\dot{P}=A(t) P+P A^{\top}(t)-P C^{\top}(t) Q(t) C(t) P+V(t)
$$

with $P(0)$ a symmetric positive definite matrix. Provided that the matrices $A(t), C(t), Q(t)$ and $V(t)$ are bounded, the existence of $\delta>0$ and $\epsilon>0$ such that $W_{V}^{A}(t, t+\delta)>\epsilon I_{n}$ and $W_{Q}^{A, C}(t, t+\delta)>\epsilon I_{n}$ for all $t \geq 0$ then ensures i) that the solution $P(t)$ to the CRE exists on $\mathbb{R}^{+}$and ii) the existence of positive numbers $p_{m}$ and $p_{M}$ such that $p_{m} I_{n} \leq P(t) \leq p_{M} I_{n}$ (see [1], for instance). In view of the previous definitions, the above mentioned properties of $W_{V}^{A}$ and $W_{Q}^{A, C}$ are automatically granted when $V(t)$ and $Q(t)$ are larger than some positive definite matrix and the pair $(A, C)$ is uniformly observable.

\section{A FRAMEWORK FOR DESIGN AND ANALYSIS OF A CLASS OF OBSERVERS}

Consider:

- a control system living in $\mathcal{B}_{r}^{n} \times \mathbb{R}^{n}$ and whose state $x=$ $\left(x_{1}^{\top}, x_{2}^{\top}\right)^{\top}$, with $x_{1} \in \mathcal{B}_{r}^{n}$ and $x_{2} \in \mathbb{R}^{n}$, evolves according to an equation of the form:

$$
\dot{x}=A\left(x_{1}, t\right) x+\left(\begin{array}{c}
u_{1} \\
u_{2}(t)
\end{array}\right)+O\left(\left|x_{1}\right|^{2}\right)+O\left(\left|x_{1}\right|\left|u_{1}\right|\right)
$$

with $\operatorname{dim}\left(u_{1}\right)=\operatorname{dim}\left(u_{2}\right)=n, A\left(x_{1}, t\right)$ a continuous matrixvalued function uniformly bounded w.r.t $t$ (and thus bounded), uniformly continuous w.r.t. the variable $x_{1}$ in $\mathcal{B}_{r}^{n}$, and such that

$$
A\left(x_{1}, t\right)=\left[\begin{array}{cc}
A_{1,1}(t) & 0_{n} \\
A_{2,1}\left(x_{1}, t\right) & A_{2,2}(t)
\end{array}\right]
$$

It is also assumed that $u_{2}(t)$ is bounded and that the solutions to this system (thus including the initial condition $x(0)$ ) belong to a compact set $\mathcal{D}$ independently of the control $u_{1}$ applied to the system.

- an "output" function $y: \mathbb{R}^{n} \times \mathbb{R}^{n} \times \mathbb{R}^{+} \rightarrow \mathbb{R}^{m}$ such that $\forall\left(x, \hat{x}_{2}, t\right) \in\left(\mathcal{B}_{r}^{n} \times \mathbb{R}^{n}\right) \times \mathbb{R}^{n} \times \mathbb{R}^{+}$:

$$
\begin{aligned}
y\left(x, \hat{x}_{2}, t\right)= & C_{1}\left(x_{1}, \hat{x}_{2}, t\right) x_{1}+C_{2}\left(x_{1}, \hat{x}_{2}, t\right) x_{2} \\
& +O\left(\left|x_{1}\right|^{2}\right)+O\left(\left|x_{1}\right|\left|x_{2}-\hat{x}_{2}\right|\right)
\end{aligned}
$$

with $C=\left[C_{1}, C_{2}\right]$ denoting a continuous matrix-valued function uniformly bounded w.r.t. the time variable $t$ and uniformly continuous w.r.t. $\left(x_{1}, \hat{x}_{2}\right)$ in a set containing $\mathcal{D}$.

- a second system interconnected with the first one and whose state $\left(\hat{x}_{2}, P\right) \in\left(\mathbb{R}^{n} \times \mathcal{S}_{+}^{2 n}\right)$ evolves according to

$$
\begin{aligned}
\dot{\hat{x}}_{2}= & A_{2,2}(t) \hat{x}_{2}+u_{2}(t) \\
& +K_{2}\left(P, x_{1}, \hat{x}_{2}, t\right)\left(y\left(x_{1}, \hat{x}_{2}, t\right)-C_{2}\left(x_{1}, \hat{x}_{2}, t\right) \hat{x}_{2}\right) \\
\dot{P}= & A\left(x_{1}, t\right) P+P A^{\top}\left(x_{1}, t\right) \\
& -P C^{\top}\left(x_{1}, \hat{x}_{2}, t\right) Q(t) C\left(x_{1}, \hat{x}_{2}, t\right) P+V(t)
\end{aligned}
$$

with $P(0)$ a symmetric positive definite matrix, $Q$ and $V$ bounded continuous symmetric positive semidefinite matrixvalued functions, and the "gain" $K$ given by

$$
\begin{aligned}
& K\left(P, x_{1}, \hat{x}_{2}, t\right)=k(t) P C^{\top}\left(x_{1}, \hat{x}_{2}, t\right) Q(t) \\
& K=\left[\begin{array}{c}
K_{1} \\
K_{2}
\end{array}\right]
\end{aligned}
$$

with $0.5 \leq k(t) \leq k_{\max }<\infty$.

\section{Remarks:}

- In this section (3) is an abstract system, but in the forthcoming developments $x_{1}$ will denote a 3-dimensional vector of coordinates characterizing the orientation error between the body orientation and the estimated orientation, and $x_{2}$ will denote a 3-dimensional vector of 
coordinates characterizing the body position. Accordingly the estimated value $\hat{x}_{1}$ of $x_{1}$ will be set equal to zero, $u_{1}$ will be a difference between the (known) body's angular velocity vector and the (calculated) angular velocity vector associated with the estimated orientation, and $u_{2}$ will be the (known) body translational velocity vector. The chosen output $y($.$) will be a vector-valued function of the$ bearing measurements, of the estimated body orientation, and of the source points positions.

- One recognizes a CRE in (6) and may interpret (5) as an observer of the state $x_{2}$ when $x_{1}$ is known.

- By setting $\hat{x}=\left(\hat{x}_{1}^{\top}, \hat{x}_{2}^{\top}\right)^{\top}$ with $\hat{x}_{1}(t)=0(\forall t \geq 0)$, (5) is equivalent to

$$
\begin{aligned}
\dot{\hat{x}}= & A\left(x_{1}, t\right) \hat{x}+\left(\begin{array}{c}
u_{1} \\
u_{2}(t)
\end{array}\right) \\
& +K\left(P, x_{1}, \hat{x}_{2}, t\right)\left(y\left(x_{1}, \hat{x}_{2}, t\right)-C\left(x_{1}, \hat{x}_{2}, t\right) \hat{x}\right)
\end{aligned}
$$

with

$$
u_{1}=-K_{1}\left(P, x_{1}, \hat{x}_{2}, t\right)\left(y\left(x_{1}, \hat{x}_{2}, t\right)-C\left(x_{1}, \hat{x}_{2}, t\right) \hat{x}\right)
$$

This writing shows the formal kinship between (5)-(6) and a standard Riccati observer -formally defined as a deterministic generalization of a Kalman filter with $k(t)$ not necessarily equal to one and matrices $Q^{-1}(t)$ and $V(t)$ not necessarily equal to noise covariance matricesapplied to a LTV system. In Section IV pose observers are derived with $x_{1}$ representing the vector part of a Rodrigues unit quaternion, and the kinship of these observers with so-called Multiplicative Extended Kalman Filters (MEKF) [13] will then appear clearly to the informed reader.

We consider two cases:

case 1: $A(t)$ does not have to be skew-symmetric, and $V(t)$ and $P(0)$ are chosen larger than some positive definite matrix. case 2: $A(t)$ is skew-symmetric (i.e. $A_{2,1}=0_{n}$, $\overline{A_{1,1}(t)}$ and $A_{2,2}(t)$ are skew-symmetric) and the constant matrix $P(t)=P(0)=\left[\begin{array}{cc}k_{1} I_{n} & 0_{n} \\ 0_{n} & k_{2} I_{n}\end{array}\right]$, with $k_{1}>0$ and $k_{2}>0$, is the chosen solution to the CRE (6) used in (5), obtained by implicitly setting $V(t)=P(0) C^{\top}\left(x_{1}(t), \hat{x}_{2}(t), t\right) Q(t) C\left(x_{1}(t), \hat{x}_{2}(t), t\right) P(0)$ $(\geq 0)$.

This latter choice of $P(t)$ is of interest to reduce the number of calculations. However, it may not yield the best observer's performance.

\section{Theorem 3.1: Set}

$$
u_{1}=-K_{1}\left(P, x_{1}, \hat{x}_{2}, t\right)\left(y\left(x_{1}, \hat{x}_{2}, t\right)-C_{2}\left(x_{1}, \hat{x}_{2}, t\right) \hat{x}_{2}\right)
$$

Define $A^{\star}(t):=A(0, t), C^{\star}(t):=C\left(0, x_{2}(t), t\right), \tilde{x}_{2}:=x_{2}-$ $\hat{x}_{2}$. If there exist $\delta>0$ and $\epsilon>0$ such that $W_{V}^{A^{\star}}(t, t+$ $\delta)>\epsilon I_{2 n}$ and $W_{Q}^{A^{\star}, C^{\star}}(t, t+\delta)>\epsilon I_{2 n}$ for all $t>0$, then $\left(x_{1}, \tilde{x}_{2}\right)=(0,0)$ is (locally) exponentially stable.

Corollary 3.2: If $Q(t)$ and $V(t)$ are larger than some positive matrix and the pair $\left(A^{\star}, C^{\star}\right)$ is uniformly observable, then $\left(x_{1}, \tilde{x}_{2}\right)=(0,0)$ is (locally) exponentially stable.

\section{Proof [Theorem 3.1]}

Define $\hat{x}=\left(\hat{x}_{1}^{\top}, \hat{x}_{2}^{\top}\right)^{\top}$ with $\hat{x}_{1}(t)=0(\forall t \geq 0)$ so that (8) holds true with $u_{1}$ given by (9). Define $\tilde{x}:=x-\hat{x}$ (= $\left.\left(x_{1}^{\top}, \tilde{x}_{2}^{\top}\right)^{\top}\right)$. Assume for the time being that $P(t), P^{-1}(t)$ and $C\left(x_{1}(t), \hat{x}_{2}(t), t\right)$ are uniformly bounded along the solutions to the systems (3) and (5)-(6) w.r.t. initial conditions taken in a small neighborhood of $\tilde{x}=0$. Using (4) and subtracting both members of (8) from the members of equality (3) yields

$$
\dot{\tilde{x}}=\left(A\left(x_{1}, t\right)-K\left(P, x_{1}, \hat{x}_{2}, t\right) C\left(x_{1}, \hat{x}_{2}, t\right)\right) \tilde{x}+O\left(|\tilde{x}|^{2}\right)
$$

This relation shows that $\tilde{x}=0$ is an equilibrium. One must now show that this equilibrium is attractive. Let us address the aforementioned two cases.

case 1: Define the positive function $L(\tilde{x}, t):=\tilde{x}^{\top} P^{-1}(t) \tilde{x}$. From this relation and the definition of $K$, and using the fact that $\frac{d}{d t} P^{-1}=-P^{-1} \dot{P} P^{-1}$, it comes that

$\dot{L}=-\tilde{x}^{\top}\left((2 k-1) C^{\top} Q C+P^{-1} V P^{-1}\right) \tilde{x}+2 \tilde{x}^{\top} P^{-1} O\left(|\tilde{x}|^{2}\right)$

Therefore, using the assumed boundedness of $P^{-1}$

$$
\dot{L}=-\tilde{x}^{\top}\left((2 k-1) C^{\top} Q C+P^{-1} V P^{-1}\right) \tilde{x}+O\left(|\tilde{x}|^{3}\right)
$$

with $O\left(|\tilde{x}|^{3}\right)=O\left(|L|^{3 / 2}\right)$. Since $V(t)$ is larger than some positive definite matrix there exists $k_{L}>0$ such that $\tilde{x}^{\top} P^{-1} V P^{-1} \tilde{x} \geq k_{L} L$. This yields

$$
\dot{L} \leq-k_{L} L+O\left(|L|^{3 / 2}\right)
$$

and, subsequently, the (local) exponential stability of $L=0$. From the definition of $L$ this in turn implies that $\tilde{x}=0$ is locally exponentially stable.

There remains to prove that if $|\tilde{x}(0)|$ is small enough then $P(t), P^{-1}(t)$ and $C\left(x_{1}(t), \hat{x}_{2}(t), t\right)$ are uniformly bounded. We already know that the boundedness of these matrices is granted when $\tilde{x}(0)=0$, as a consequence of the assumptions made on $W_{V}^{A^{\star}}$ and $W_{Q}^{A^{\star}, C^{\star}}$.

Let $p_{M}(t)$ (resp. $p_{m}(t)$ ) denote the largest (resp. smallest) eigenvalue of $P(t)$. Since the matrices $A\left(x_{1}(t), t\right)$ and $V(t)$ are bounded, $p_{M}(t)$ cannot grow faster than exponentially with $t$, whereas $p_{m}(t)$ is always positive but may tend to zero (see Appendix of [1]). These properties of the CRE hold independently of $|\tilde{x}(0)|$ and $Q(t)$. Combining this maximum increase rate of $p_{M}(t)$ with the boundedness of $C^{\star}(t)$ and the existence of $\gamma_{c}>0$ such that $\left|C\left(x_{1}, \hat{x}_{2}, t\right)-C^{\star}(t)\right|<\gamma_{c}|\tilde{x}|$ (by the assumed property of uniform continuity of $\left.C\left(x_{1}, \hat{x}_{2}, t\right)\right)$ one deduces, by inspection of the equation of evolution of the error $\tilde{x}$ and the definition of the gain $K\left(P, x_{1}, \hat{x}_{2}, t\right)$, the existence of three positive exponentially increasing functions $\beta_{1}(t), \beta_{2}(t)$ and $\beta_{1}(t)$ such that, given any $t_{0}>\delta$

$$
\frac{d}{d t}|\tilde{x}| \leq \beta_{1}\left(t_{0}\right)|\tilde{x}|+\beta_{2}\left(t_{0}\right)|\tilde{x}|^{2}+\beta_{3}\left(t_{0}\right)|\tilde{x}|^{3} ; t \in\left[0, t_{0}\right]
$$

This relation in turn implies that given any $\epsilon_{1}>0$, there exists a positive number $\alpha\left(\epsilon_{1}, t_{0}\right)$ such that $|\tilde{x}(0)|<\alpha\left(\epsilon_{1}, t_{0}\right)$ implies that $|\tilde{x}(t)| \leq \epsilon_{1}$ for $t \in\left[0, t_{0}\right]$. Therefore, choosing $\epsilon_{1}$ small enough and using again the uniform continuity of the matrix-valued functions $A$ and $C$, ensures that $\left|W_{V}^{A}(t, t+\delta)-W_{V}^{A^{\star}}(t, t+\delta)\right|$ and 
$\left|W_{Q}^{A, C}(t, t+\delta)-W_{Q}^{A^{\star}, C^{\star}}(t, t+\delta)\right|$ are smaller than $\epsilon / 2$ for $t \in\left[0, t_{0}-\delta\right]$. This in turn implies that $W_{V}^{A}(t, t+\delta)>\epsilon / 2$ and $W_{Q}^{A, C}(t, t+\delta)>\epsilon / 2$ for $t \in\left[0, t_{0}-\delta\right]$ and, subsequently, that $p_{M}(t)$ (resp. $p_{m}(t)$ ) is in fact upper-bounded (resp. lower-bounded) on $\left[0, t_{0}\right]$ by a number $p_{M, \epsilon}\left(\right.$ resp. $\left.p_{m, \epsilon}\right)$ that depends on $\epsilon$, but not on $t_{0}$. A lower bound of the gain $k_{L}$ in (11), valid on the time interval $\left[0, t_{0}\right]$ when $|\tilde{x}(0)|<\alpha\left(\epsilon_{1}, t_{0}\right)$, is then equal to $\frac{p_{m, \epsilon}}{p_{M, \epsilon}^{2}} v_{m}$ with $v_{m}$ denoting the (positive) ultimate lower bound of the eigenvalues of $V(t)$. From (11) we then deduce that $L(t) \leq L(0) \exp \left(-0.5 \frac{p_{m, \epsilon}}{p_{M, \epsilon}^{2}} v_{m} t\right)$ on the time interval $\left[0, t_{0}\right]$ provided that $\alpha\left(\epsilon_{1}, t_{0}\right)$ is chosen small enough. Since $L(0) \leq|\tilde{x}(0)|^{2} / p_{m, \epsilon}$ and $L(t) \geq|\tilde{x}(t)|^{2} / p_{M, \epsilon}$ on this time interval it also comes that $\left|\tilde{x}\left(t_{0}\right)\right| \leq \sqrt{\frac{p_{M, \epsilon}}{p_{m, \epsilon}} \exp \left(-0.5 \frac{p_{m, \epsilon}}{p_{M, \epsilon}^{2}} v_{m} t_{0}\right)}|\tilde{x}(0)|$. Therefore, there exists a value of $t_{0}$ such that $\left|\tilde{x}\left(t_{0}\right)\right| \leq|\tilde{x}(0)|$, provided that $\alpha\left(\epsilon_{1}, t_{0}\right)$ (the considered upper bound of $\left.|\tilde{x}(0)|\right)$ is chosen small enough. From there it only remains to repeat the same arguments with $\tilde{x}\left(t_{0}\right)$ taken as the new initial condition to establish that the previous bounds on $|\tilde{x}(t)|$ (and thus $\left.\mid C\left(x_{1}(t), \hat{x}_{2}(t), t\right)\right)$ and on $P(t)$ continue to hold on the time interval $\left[t_{0}, 2 t_{0}\right]$, provided that $|\tilde{x}(0)|$ is chosen small enough. Repeating this procedure for all time intervals $\left[j t_{0},(j+1) t_{0}\right]$ $(j \in \mathbb{N})$ establishes the announced boundedness results on $[0,+\infty)$.

case 2: Note that in this case $W_{Q}^{A^{\star}, C^{\star}}(t, t+\delta)=W_{V}^{A^{\star}}(t, t+\delta)$. Also, by assumption

$$
A\left(x_{1}(t), t\right)=\left[\begin{array}{cc}
-S_{1}(t) & 0_{n} \\
0_{n} & -S_{2}(t)
\end{array}\right]
$$

with $S_{1}(t)$ and $S_{2}(t)$ denoting skew-symmetric matrices. Define the rotation matrices $R_{1}(t)$ and $R_{2}(t)$ in $S O(n)$ solutions to $\dot{R}_{i}=R_{i} S_{i}(i=1,2)$ with $R_{1}(0)=R_{2}(0)=I_{n}$. Then

$$
\Phi(t, s)=\left[\begin{array}{cc}
R_{1}^{\top}(t) R_{1}(s) & 0_{n} \\
0_{n} & R_{2}^{\top}(t) R_{2}(s)
\end{array}\right]
$$

Define $\bar{x}(t):=\left[\begin{array}{l}R_{1}(t) \tilde{x}_{1}(t) / \sqrt{k_{1}} \\ R_{2}(t) \tilde{x}_{2}(t) / \sqrt{k_{2}}\end{array}\right]$. From (10) and the fact that $K C=k P C^{\top} Q C$ one gets

$$
\begin{aligned}
\dot{\bar{x}}= & -k\left[\begin{array}{cc}
\sqrt{k_{1}} I_{n} & 0_{n} \\
0_{n} & \sqrt{k_{2}} I_{n}
\end{array}\right] B(t)\left[\begin{array}{cc}
\sqrt{k_{1}} I_{n} & 0_{n} \\
0_{n} & \sqrt{k_{2}} I_{n}
\end{array}\right] \bar{x} \\
& +O\left(|\bar{x}|^{2}\right)
\end{aligned}
$$

with

$$
\begin{aligned}
B(t) & :=\bar{R}(t) C^{\top}\left(x_{1}(t), \hat{x}_{2}(t), t\right) Q(t) C\left(x_{1}(t), \hat{x}_{2}(t), t\right) \bar{R}^{\top}(t) \\
\bar{R}(t) & =\left[\begin{array}{cc}
R_{1}(t) & 0_{n} \\
0_{n} & R_{2}(t)
\end{array}\right]
\end{aligned}
$$

Using the uniform continuity property of $C$ and the boundedness of $Q(t)$, relation (12) also holds when replacing $B(t)$ by $B^{\star}(t)$ with

$$
B^{\star}(t):=\bar{R}(t) C^{\star \top}(t) Q(t) C^{\star}(t) \bar{R}^{\top}(t)
$$

By a slight generalization of Lemma 5 in [14] one deduces that $\bar{x}=0$, and thus $\tilde{x}=0$, are locally exponentially stable provided that there exist $\delta>0$ and $\bar{\epsilon}>0$ such that $\frac{1}{\delta} \int_{t}^{t+\delta} B^{\star}(s) d s>\bar{\epsilon} I_{2 n \times 2 n}(\forall t \geq 0)$. This last inequality is clearly satisfied with $\bar{\epsilon}=\epsilon$ since

$$
\frac{1}{\delta} \int_{t}^{t+\delta} B^{\star}(s) d s=\bar{R}^{\top}(t) W_{Q}^{A^{\star}, C^{\star}}(t, t+\delta) \bar{R}(t)
$$

\section{Application to POSE ESTIMATION FROM BEARING MEASUREMENTS}

\section{A. Problem statement}

Let us start with some notation:

- $\mathcal{F}$ is an inertial frame.

- $\mathcal{B}$ is a frame attached to a possibly moving body whose pose is estimated. Its origin is the point $C$. This point may, for instance, be the optic centre of a camera.

- $R(t)$ is the rotation matrix characterizing the orientation at time $t$ of $\mathcal{B}$ w.r.t. $\mathcal{F}$.

- $\omega(t)$ is the vector of coordinates at time $t$ of the instantaneous angular velocity of $\mathcal{B}$ expressed in the basis of $\mathcal{B}$, i.e. $\dot{R}(t)=R(t) S(\omega(t))$

- $p(t)$ (resp. $\bar{p}(t)$ ) is the vector of coordinates at time $t$ of the position of $C$ w.r.t. $\mathcal{F}$ expressed in the basis of $\mathcal{F}$ (resp. $\mathcal{B})$. Therefore $\bar{p}=R^{\top} p$.

- $v(t)$ (resp. $\bar{v}(t)$ ) is the vector of coordinates at time $t$ of the velocity of $C$ w.r.t. $\mathcal{F}$ expressed in the basis of $\mathcal{F}$ (resp. $\mathcal{B})$. Therefore: $\dot{p}=v, \bar{v}=R^{\top} v$, and $\dot{\bar{p}}=-S(\omega) \bar{p}+\bar{v}$. We assume that the motion of $\mathcal{B}$ keeps the distance $|p(t)|$ between the origins of $\mathcal{F}$ and $\mathcal{B}$ bounded.

- $z_{i}(i=1, \ldots, l)$ is the vector of coordinates of the position of $i$ th source point $P_{i}$ w.r.t. $\mathcal{F}$ expressed in the basis of this frame.

- $d_{i}(t):=R^{\top}(t)\left(p(t)-z_{i}\right) /\left|p(t)-z_{i}\right|(i=1, \ldots, l)$ is the unitary vector of coordinates characterizing the direction (or bearing) of $\overrightarrow{P_{i} C}$ expressed in the basis of $\mathcal{B}$.

- $\hat{p}(t)$ (resp. $\hat{\bar{p}}(t)$ ) is an estimate at time $t$ of $p(t)$ (resp. $\bar{p}(t)$ ). - $\hat{R}(t)$ is an estimate at time $t$ of $R(t)$. This is also a rotation matrix and the corresponding angular velocity vector expressed in the basis of $\mathcal{B}$ is denoted as $\hat{\omega}(t)$, i.e. $\dot{\hat{R}}=\hat{R} S(\hat{\omega})$. Given an initial condition $\hat{R}(0)$ and $\hat{\omega}(t)$, $\hat{R}(t)$ can be calculated by numerical integration of the latter equality. This calculation may also be performed by using a unitary quaternion associated with $\hat{R}(t)$.

The problem at hand is to produce an on-line estimation of $p(t)$ and $R(t)$ given the measurement of the angular velocity $\omega(t)$, the knowledge of the $l$ source point positions $z_{i}$, their directions $d_{i}(t)$, and either the inertial velocity vector $v(t)$ or the mobile velocity vector $\bar{v}(t)$. We will see that it is important to distinguish between these two velocity measurement cases in relation to observability conditions under which the estimated pose $(\hat{p}(t), \hat{R}(t))$ can robustly converge to the actual body pose $(p(t), R(t))$. We will first address the "mobile velocity measurement case" and then the more involved, but also more versatile, "inertial velocity measurement case". 
Prior to deriving specific pose observers it is useful to recall a few facts about minimal parametrizations of the group of rotations, first order approximations of nonlinear functions and also kinematic relations repeatedly called for in the forthcoming developments.

\section{Facts:}

- There are infinitely many (local) minimal paramerizations of the three-dimensional Lie group $S O(3)$ of rotation matrices. Common ones are the vector part of a Hamilton or of a Rodrigues unit quaternion, Euler angles, Cardan (or Tait-Bryan) angles, etc. Theorem 3.1 can be adapted to any of these parametrizations to derive as many different pose observers endowed with similar local properties. We will favour here the vector part of a Rodrigues unit quaternion due to its simplicity of use and the fact that it provides a regular representation of rotation matrices for rotation angles up to $\pi$ (excluded), by contrast with Euler angles that have representation singularities at $\pi / 2$. The choice of quaternions lends itself to several possible parametrizations. We will consider two of them in this work.

The first one involves the unit quaternion $\Lambda=\left(\lambda_{0}, \lambda\right)$, with $\lambda \in \mathcal{B}_{1}^{3}$ (resp. $\lambda_{0} \in \mathcal{B}_{1}^{1}$ ) denoting the vector (resp. scalar) part of the quaternion, associated with the "error" rotation matrix $\tilde{R}:=R \hat{R}^{\top}$. Rodrigues formula relating $\Lambda$ to $\tilde{R}$ is

$$
\tilde{R}=I_{3}+2 S(\lambda)\left(\lambda_{0} I_{3}+S(\lambda)\right)
$$

Define $\tilde{\omega}:=\omega-\hat{\omega}$, we have the following kinematic relations

$$
\dot{\tilde{R}}=\tilde{R} S(\hat{R} \tilde{\omega}) ; \quad\left\{\begin{array}{l}
\dot{\lambda}_{0}=-0.5(\hat{R} \tilde{\omega})^{\top} \lambda \\
\dot{\lambda}=0.5 \hat{R} \tilde{\omega} \lambda_{0}+0.5 S(\hat{R} \tilde{\omega}) \lambda
\end{array}\right.
$$

so that, using the fact that $\lambda_{0}=1-|\lambda|^{2}$

$$
\left\{\begin{array}{l}
\tilde{R}=I_{3}+2 S(\lambda)+O\left(|\lambda|^{2}\right) \\
2 \dot{\lambda}=\hat{R} \tilde{\omega}+O(|\lambda||\tilde{\omega}|)
\end{array}\right.
$$

A second possible parametrization involves the unit quaternion $\bar{\Lambda}=\left(\bar{\lambda}_{0}, \bar{\lambda}\right)$ associated with the "error" rotation matrix $\overline{\tilde{R}}:=\hat{R}^{\top} R$. Rodigues formula relating $\bar{\Lambda}$ to $\overline{\tilde{R}}$ is

$$
\overline{\tilde{R}}=I_{3}+2 S(\bar{\lambda})\left(\bar{\lambda}_{0} I_{3}+S(\bar{\lambda})\right)
$$

Define $\overline{\tilde{\omega}}:=\omega-\overline{\tilde{R}}^{\top} \hat{\omega}$, we have the following kinematic relations

$$
\dot{\tilde{\tilde{R}}}=\overline{\tilde{R}} S(\overline{\tilde{\omega}}) ; \quad\left\{\begin{array}{l}
\dot{\bar{\lambda}}_{0}=-0.5 \overline{\tilde{\omega}}^{\top} \bar{\lambda} \\
\dot{\bar{\lambda}}=0.5 \overline{\tilde{\omega}} \bar{\lambda}_{0}+0.5 S(\overline{\tilde{\omega}}) \bar{\lambda}
\end{array}\right.
$$

so that, using the the fact that $\bar{\lambda}_{0}=1-|\bar{\lambda}|^{2}$

$$
\left\{\begin{array}{l}
\overline{\tilde{R}}=I_{3}+2 S(\bar{\lambda})+O\left(|\bar{\lambda}|^{2}\right) \\
2 \dot{\bar{\lambda}}=\tilde{\omega}-S(\omega)(2 \bar{\lambda})+O(|\bar{\lambda}||\tilde{\omega}|)+O\left(|\omega||\bar{\lambda}|^{2}\right)
\end{array}\right.
$$

Since $|\omega(t)|$ is bounded by assumption, one can also write

$$
2 \dot{\bar{\lambda}}=\tilde{\omega}-S(\omega)(2 \bar{\lambda})+O(|\bar{\lambda}||\tilde{\omega}|)+O\left(|\bar{\lambda}|^{2}\right)
$$

- It is common knowledge that first order approximations of a nonlinear system $\dot{x}=f(x, t)$ about a current stateestimate $\hat{x}$ can be used to derive state observers for this system. The classically invoked linear approximation $\dot{x}=\frac{\delta}{\delta x} f(\hat{x}, t)(x-\hat{x})$ is only one among infinitely many first order approximations. For instance any system $\dot{x}=\frac{\delta}{\delta x} f(\hat{x}, t)(x-\hat{x})+O\left(|x-\hat{x}|^{2}\right)$ is also a first order approximation about $\hat{x}$ in the sense that the error committed in the right-hand side of the equality is still a $O\left(|x-\hat{x}|^{2}\right)$. This remark serves to point out that the observers derived further in the paper exploit first order approximations that are not necessarily linear approximations, and that several other variants of these observers can be obtained by just considering different first order approximations.

To complement these facts we recall that there are infinitely many ways to minimally parametrize the pose of a body in 3D-space and that each of these parametrizations yields specific linear and first-order approximations. The observer design methodology here considered can be adapted to any such parametrization and it can potentially produce infinitely many different observers having in common the same local stability and convergence properties. Let us for instance mention the four parametrizations $(\lambda, p),(\bar{\lambda}, p),(\lambda, \bar{p})$ and $(\bar{\lambda}, \bar{p})$ for which Theorem 3.1 applies to derive and analyse different pose Riccati observers endowed with similar local properties. In what follows this possibility is illustrated by considering two of these parametrizations, namely $(\bar{\lambda}, \bar{p})$ in the case of mobile velocity measurements and $(\lambda, p)$ in the case of inertial velocity measurements, and by simulating the observers associated with them.

\section{B. Mobile velocity measurement}

From now on $R(t)$ is taken as a function of time so that $\hat{R}=\tilde{R}^{\top} R=R \overline{\tilde{R}}$ can be seen either as a function of $t$ and $\lambda$, or a function of $t$ and $\bar{\lambda}$.

1) Observer based on the parametrization $(\bar{\lambda}, \bar{p})$ : One has

$$
\left\{\begin{array}{l}
2 \dot{\bar{\lambda}}=-S(\omega)(2 \bar{\lambda})+\tilde{\omega}+O(|\bar{\lambda}||\tilde{\omega}|)+O\left(|\omega||\bar{\lambda}|^{2}\right) \\
\dot{\bar{p}}=-S(\omega) \bar{p}+\bar{v}
\end{array}\right.
$$

Setting $x_{1}=2 \bar{\lambda}, x_{2}=\bar{p}$ and using the assumed boundedness of $|\omega(t)|$, one obtains the system equation (3) with

$$
A_{1,1}(t)=A_{2,2}(t)=-S(\omega(t)), A_{2,1}=0_{3}, u_{1}=\tilde{\omega}, u_{2}=\bar{v}
$$

Concerning the output function $y$, one has for $i=1, \ldots, l$

$$
\begin{aligned}
\left|p-z_{i}\right| d_{i} & =\bar{p}-R^{\top} z_{i} \\
& =\bar{p}-\overline{\tilde{R}}^{\top} \hat{R}^{\top} z_{i} \\
& =\bar{p}-\hat{R}^{\top} z_{i}-\left(\overline{\tilde{R}}^{\top}-I_{3}\right) \hat{R}^{\top} z_{i} \\
& =\bar{p}-\hat{R}^{\top} z_{i}+S(2 \bar{\lambda}) \hat{R}^{\top} z_{i}+O\left(\left|z_{i} \| \bar{\lambda}\right|^{2}\right) \\
& =\bar{p}-\hat{R}^{\top} z_{i}-S\left(\hat{R}^{\top} z_{i}\right)(2 \bar{\lambda})+O\left(\left|z_{i}\right||\bar{\lambda}|^{2}\right)
\end{aligned}
$$

so that, by setting again $y_{i}(\bar{\lambda}, \bar{p}, t):=\Pi_{d_{i}(t)} \hat{R}^{\top} z_{i}$ and using the identity $\Pi_{d_{i}} d_{i}=0$, one obtains

$$
y_{i}(\bar{\lambda}, \bar{p}, t)=-\Pi_{d_{i}(t)} S\left(\hat{R}^{\top} z_{i}\right)(2 \bar{\lambda})+\Pi_{d_{i}(t)} \bar{p}+O\left(|\lambda|^{2}\right)
$$


Therefore, defining $y:=\left(y_{1}^{\top}, \ldots, y_{l}^{\top}\right)^{\top}$ one obtains the output equation (4) with

$$
\begin{gathered}
C_{1}\left(x_{1}, \hat{x}_{2}, t\right)=\left[\begin{array}{c}
-\Pi_{d_{1}(t)} S\left(\hat{R}^{\top} z_{1}\right) \\
\vdots \\
-\Pi_{d_{l}(t)} S\left(\hat{R}^{\top} z_{l}\right)
\end{array}\right] \\
C_{2}\left(x_{1}, t\right)=\left[\begin{array}{c}
\Pi_{d_{1}(t)} \\
\vdots \\
\Pi_{d_{l}(t)}
\end{array}\right]
\end{gathered}
$$

The corresponding Riccati observer giving the expressions of $\hat{\omega}$ and $\dot{\bar{p}}$ (that are used to compute $\hat{R}(t)$ and $\hat{\bar{p}}(t)$ via numerical integration) is then given by (5)-(7) and (9).

Remark: The observer derived previously uses outputs $y_{i}=$ $\Pi_{d_{i}} \hat{R}^{\top} z_{i}$. But other outputs may also be used. For instance, defining $\hat{d}_{i}:=\hat{R}^{\top}\left(\hat{p}-z_{i}\right) /\left|\hat{p}-z_{i}\right|$ and using the fact that $\Pi_{\hat{d}_{i}} d_{i}=O(|\lambda|)+O(|\tilde{p}|)$ provided that $\left|p(t)-z_{i}\right|>\mu>0$ for all $t$, one can set $y_{i}:=\left|\hat{p}-z_{i}\right| \Pi_{\hat{d}_{i}} d_{i}+\Pi_{\hat{d}_{i}} R^{\top} z_{i}$ so that $y_{i}=C_{1}(2 \lambda)+C_{2} p+O\left(|\lambda|^{2}+|\tilde{p}|^{2}\right)$ with $C_{1}=\left|\hat{p}-z_{i}\right| S\left(\hat{d}_{i}\right) \hat{R}^{\top}$ (using the fact that $\Pi_{x /|x|} S(x)=S(x)$ ) and $C_{2}=\Pi_{\hat{d}_{i}} \hat{R}^{\top}$.

Similarly, defining $\hat{\bar{d}}_{i}=\left(\hat{\bar{p}}-\hat{R}^{\top} z_{i}\right) /\left|\hat{\bar{p}}-\hat{R}^{\top} z_{i}\right|$ and using the fact that $\Pi_{\hat{\bar{d}}_{i}} d_{i}=O(|\bar{\lambda}|)+O(|\overline{\tilde{p}}|)$ provided that $\left|p(t)-z_{i}\right|>\mu>0$ for all $t$, one can set $y_{i}:=\left|\hat{\bar{p}}-\hat{R}^{\top} z_{i}\right| \Pi_{\hat{\bar{d}}_{i}} d_{i}+\Pi_{\hat{\bar{d}}_{i}} \hat{R}^{\top} z_{i}$ so that $y_{i}=C_{1}(2 \bar{\lambda})+C_{2} \bar{p}+O\left(|\bar{\lambda}|^{2}+|\overline{\tilde{p}}|^{2}\right)$ with $C_{1}=-\Pi_{\hat{\bar{d}}_{i}} S\left(\hat{R}^{\top} z_{i}\right)$ and $C_{2}=\Pi_{\hat{d}_{i}}$

As already mentioned all previously evoked parametrizations yield Riccati observers sharing the same local properties. Determination and comparison of the associated domains of attraction in order to eventually work out to some type of efficiency ranking between these parametrizations are legitimate (but difficult) questions. They are not within the scope of the present work but they may motivate future studies. Note however that these issues are not necessarily critical in practice due to the existence, in a certain number of cases, of complementary algebraic solutions that can provide good initial estimates of the body pose [2].

2) Observability issues: Once the general expression of the observer is obtained it matters to determine conditions whose satisfaction ensures that the observer is exponentially stable, i.e. that zero estimation errors are uniformly exponentially stable. As pointed out in Theorem 3.1 and Corollary 3.2 uniform observability of the associated pair $\left(A^{\star}(t), C^{\star}(t)\right)$ suffices, provided that the matrix $Q(t)$ entering the CRE is chosen larger than some positive matrix, and that the matrix $V(t)$ is either larger than some positive matrix or such that $W_{V}^{A^{\star}}(t, t+$ $\delta)>\epsilon I_{6}$. With $\Phi^{\star}(t, s)$ denoting the transition matrix associated with $A^{\star}(t)$, the strict positivity of the observability Gram$\operatorname{mian} W^{\star}(t, t+\delta):=\int_{t}^{t+\delta} \Phi^{\star \top}(s, t) C^{\star \top}(s) C^{\star}(s) \Phi^{\star}(s, t) d s$ for some $\delta>0$ and all $t \geq 0$ is thus the central property in this respect. When it is only semi-definite positive the condition number of the matrix $P(t)$ solution to the CRE generally diverges when the origin of the system $\dot{x}=A^{\star}(t) x$ is not exponentially stable (as in the pose estimation case here considered) and the estimation errors do not uniformly exponentially converge to zero. We show next that the nonsatisfaction of this property depends essentially upon the number of source points and, in the specific case of three source points, upon the body position relatively to the socalled "danger cylinder" well known of persons familiarized with the Perspective-n-Point (PnP) problem [2], [6].

One can verify that all observers considered so far share the same observability Grammian $W^{\star}(t, t+\delta)$, modulo pre and post multiplication by regular matrices $B(t)$ and $B(t)^{\top}$ whose singular values are bounded from below and above by positive numbers and thus do not affect the uniform observability criterion. This was expected since all these observers address the same estimation problem. Let us then consider, for example, the observer associated with the parametrization $(\bar{\lambda}, \bar{p})$ and examine the observability Grammian according to the number and disposition of the source points and the position/motion of the point $C$ relatively to the source points. In the case of the parametrization $(\bar{\lambda}, \bar{p})$ one has

$$
\begin{aligned}
A^{\star}(t) & =\left[\begin{array}{cc}
-S(\omega(t)) & 0_{3} \\
0_{3} & -S(\omega(t))
\end{array}\right] \\
C^{\star}(t) & =\left[\begin{array}{cc}
-\Pi_{d_{1}(t)} S\left(R^{\top}(t) z_{1}\right) & \Pi_{d_{1}(t)} \\
\vdots & \vdots \\
-\Pi_{d_{l}(t)} S\left(R^{\top}(t) z_{l}\right) & \Pi_{d_{l}(t)}
\end{array}\right]
\end{aligned}
$$

and thus

$$
\Phi^{\star}(s, t)=\left[\begin{array}{cc}
R(s)^{\top} R(t) & 0_{3} \\
0_{3} & R(s)^{\top} R(t)
\end{array}\right]
$$

Define

$$
\begin{aligned}
& \stackrel{\circ}{R}(t):=\left[\begin{array}{cc}
R(t) & 0_{3} \\
0_{3} & R(t)
\end{array}\right] \\
& \Delta(s, t):=\Phi^{\star \top}(s, t) C^{\star \top}(s) C^{\star}(s) \Phi^{\star}(s, t)= \\
& \stackrel{\circ}{R}^{\top}(t) \sum_{i=1}^{l}\left[\begin{array}{c}
S\left(z_{i}\right) \\
I_{3}
\end{array}\right] \Pi_{d_{i}^{\mathcal{F}}(s)}\left[\begin{array}{ll}
S^{\top}\left(z_{i}\right) & I_{3}
\end{array}\right] \stackrel{\circ}{R}(t)
\end{aligned}
$$

with $d_{i}^{\mathcal{F}}(t):=\left(p(t)-z_{i}\right) /\left|p(t)-z_{i}\right|$ denoting the direction of the ith source point expressed in the basis of the inertial frame. The observability Grammian is thus never positive on any time interval if and only if there exists a non-zero vector $w \in \mathbb{R}^{6}$ such that

$$
\Pi_{d_{i}^{\mathcal{F}}(t)}\left[S^{\top}\left(z_{i}\right) \quad I_{3}\right] w=0, \forall i \in\{1, \ldots, l\}, \forall t \geq 0
$$

This equality characterizes body position trajectories along which the body pose is not uniformly observable on any time interval.

\section{Aligned source points}

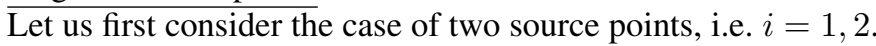
To satisfy the equality (16) it sufices that

$$
\left[\begin{array}{ll}
S^{\top}\left(z_{1}\right) & I_{3} \\
S^{\top}\left(z_{2}\right) & I_{3}
\end{array}\right] w=0
$$

One easily verifies that any vectors $w=\alpha\left[\begin{array}{c}z_{2}-z_{1} \\ z_{1} \times z_{2}\end{array}\right]$, $\alpha \in \mathbb{R}-\{0\}$, is a solution to this equation. Moreover there 
is no other solution because the kernel of the matrix that $w$ multiplies is one-dimensional.

By a simple extension of the previous proof, one shows that if all source points are aligned (independently of their number) then the observability Grammian is never positive whatever the position and motion of $C$.

\section{Three non-aligned source points}

In this case the satisfaction of (16) is equivalent to showing the existence of $(w, \stackrel{\circ}{w}(t))$, with $\stackrel{w}{(t)}(t) \mathbb{R}^{3}$ such that, for all $t \geq 0$

$$
\left[\begin{array}{ll}
M_{1} & M_{2}(t)
\end{array}\right]\left[\begin{array}{c}
w \\
\stackrel{w}{w}(t)
\end{array}\right]=0
$$

with

$$
\begin{aligned}
& M_{1}:=\left[\begin{array}{cc}
S^{\top}\left(z_{1}\right) & I_{3} \\
S^{\top}\left(z_{2}\right) & I_{3} \\
S^{\top}\left(z_{3}\right) & I_{3}
\end{array}\right] \\
& M_{2}(t):=\left[\begin{array}{ccc}
p(t)-z_{1} & 0_{3 \times 1} & 0_{3 \times 1} \\
0_{3 \times 1} & p(t)-z_{2} & 0_{3 \times 1} \\
0_{3 \times 1} & 0_{3 \times 1} & p(t)-z_{3}
\end{array}\right]
\end{aligned}
$$

We already know that, if such a solution exists, $\stackrel{w}{w}(t)$ cannot be identically equal to zero because the sub-matrix $M_{1}$ is of full rank equal to six. Now, forming the time derivative of both members of the equality (18) yields $\frac{d}{d t}\left(p(t)-z_{i}\right)=-\left(p(t)-z_{i}\right)\left(\frac{d}{d t} \stackrel{\circ}{w}_{i}(t) / \stackrel{\circ}{w}_{i}(t)\right), i=1,2,3$, with $\stackrel{\circ}{w}_{i}$ denoting the ith component of $\stackrel{\leftrightarrow}{w}$. If the point $C$ moves, i.e. $v(t) \neq 0$, the simultaneous satisfaction of these three equalities has implications namely i) the point $C$ must move along a straight line, and ii) this line has to pass through one of the source points. Let us assume, for instance that this point is $P_{3}$ and that the direction of motion of $C$ is given by a constant unit vector denoted as $\mu$, i.e. $p(t)=z_{3}+a(t) \mu$ with $a(t) \in \mathbb{R}$. Then the three equalities are satisfied with $\stackrel{\circ}{w}_{i}(t)=\frac{d}{d t} \stackrel{\circ}{w}_{i}(t)=0$ for $i=1,2$, which in turn implies, in view of (18), that (17) is satisfied and thus that $w=\alpha\left[\begin{array}{l}z_{2}-z_{1} \\ z_{1} \times z_{2}\end{array}\right]$. The third equation of (18) then yields $\alpha\left(z_{3} \times z_{2}+z_{1} \times z_{3}+z_{1} \times z_{2}\right)+a(t) \stackrel{\leftrightarrow}{w}_{3}(t) \mu=0$. This tells us that $\mu$ is orthogonal to the plane containing the three source points. Repeating the same argument for $P_{1}$ and $P_{2}$ one deduces that any motion of $C$ along a straight line orthogonal to the source points plane and passing through a source point forbids uniform observability of the body pose.

Let us now examine the static case when $C$ is motionless. The $(9 \times 9)$ matrix in the left-hand side of the equality (18) is then constant and one looks for $p$ that renders this matrix singular. Via classical lines and columns manipulations one easily verifies that this matrix is singular if and only if the the following $(6 \times 6)$ matrix

$$
D(p):=\left[\begin{array}{cccc}
S^{\top}\left(z_{2}-z_{1}\right) & z_{1}-z_{2} & p-z_{2} & 0_{3 \times 1} \\
S^{\top}\left(z_{3}-z_{1}\right) & z_{1}-z_{3} & 0_{3 \times 1} & p-z_{3}
\end{array}\right]
$$

is itself singular, i.e. if and only if $\operatorname{det}(D(p))=0$. We claim that this latter equation is nothing else than the equation of the so-called danger cylinder [2], [6], i.e. the circular cylinder generated by the circle passing through the three source points and whose axis is orthogonal to the plane containing the source points. Indeed, via a change of coordinates and scaling one can arbitrarily set $z_{1}=[0,0,0]^{\top}, z_{2}=[1,0,0]^{\top}$ and $z_{3}=[a, b, 0]^{\top}(b \neq 0)$, and then easily verify that $\operatorname{det}(D(p))=p_{1}^{2}+p_{2}^{2}-p_{1}+\left(\frac{a(1-a)}{b}-b\right) p_{2}$, with $p_{i}$ denoting the $i$ th component of $p$.

To summarize, we have shown that the body pose is not uniformly observable when $C$ is motionless on the danger cylinder, or when $C$ moves along one of the three straight lines belonging to this cylinder and passing through a source point. If $C$ moves on the danger cylinder, but not along one of these three lines, then the body pose is uniformly observable under weak complementary conditions such as $\int_{t}^{t+\delta}\left|\Pi_{\eta} v(s)\right| d s>\epsilon>0, \forall t \geq 0$, with $\eta$ denoting a unit vector orthogonal to the source points plane. If $C$ is fixed, but not on the danger cylinder, or moves without approaching the danger cylinder, or crosses this cylinder with a non-vanishing transversal velocity, uniform observability is also granted. Therefore, without minimizing the existence and practical significance of the particular trajectories of $C$ for which the body pose is not observable, one may assert that uniform observability, and thus uniform exponential stability of the observers derived previously, are "generically" granted in the case of three non-aligned source points.

\section{Four and more non-aligned source points}

In the case of four source points (16) is equivalent to the existence of $(w, \stackrel{\circ}{w}(t))$, with $w \in \mathbb{R}^{6}-\{0\}$ and $\stackrel{\leftrightarrow}{w}(t) \in \mathbb{R}^{4}$, such that for all $t \geq 0$

$$
\left[\begin{array}{ll}
M_{1} & M_{2}(t)
\end{array}\right]\left[\begin{array}{c}
w \\
\stackrel{w}{(}(t)
\end{array}\right]=0
$$

with

$$
\begin{aligned}
& M_{1}:=\left[\begin{array}{cc}
S^{\top}\left(z_{1}\right) & I_{3} \\
S^{\top}\left(z_{2}\right) & I_{3} \\
S^{\top}\left(z_{3}\right) & I_{3} \\
S^{\top}\left(z_{4}\right) & I_{3}
\end{array}\right] \\
& M_{2}(t):=\left[\begin{array}{cccc}
p(t)-z_{1} & 0_{3 \times 1} & 0_{3 \times 1} & 0_{3 \times 1} \\
0_{3 \times 1} & p(t)-z_{2} & 0_{3 \times 1} & 0_{3 \times 1} \\
0_{3 \times 1} & 0_{3 \times 1} & p(t)-z_{3} & 0_{3 \times 1} \\
0_{3 \times 1} & 0_{3 \times 1} & 0_{3 \times 1} & p(t)-z_{4}
\end{array}\right]
\end{aligned}
$$

By differentiating this equation w.r.t time and using the fact the sub-matrix $M_{1}$ is of full rank equal to six, one shows that this equation has no solution when $C$ moves, i.e. when $v(t) \neq 0$ on some time interval.

If the point $C$ is motionless one may arbitrarily choose it as the origin of the inertial frame, i.e. set $p=[0,0,0]^{\top}$. Relation (16) is then equivalent to requiring that all $z_{i}(i \in\{1, \ldots, l\})$ are solutions to the equation

$$
\Pi_{\frac{z}{|z|}}\left[S(z) \quad I_{3}\right] w=0
$$

or, equivalently, are solutions to

$$
z \times w_{1}+\Pi_{\frac{z}{|z|}} w_{2}=0
$$

for some $w=\left(w_{1}^{\top}, w_{2}^{\top}\right)^{\top}$. If $w_{1}=[0,0,0]^{\top}$ and $w_{2}$ is 


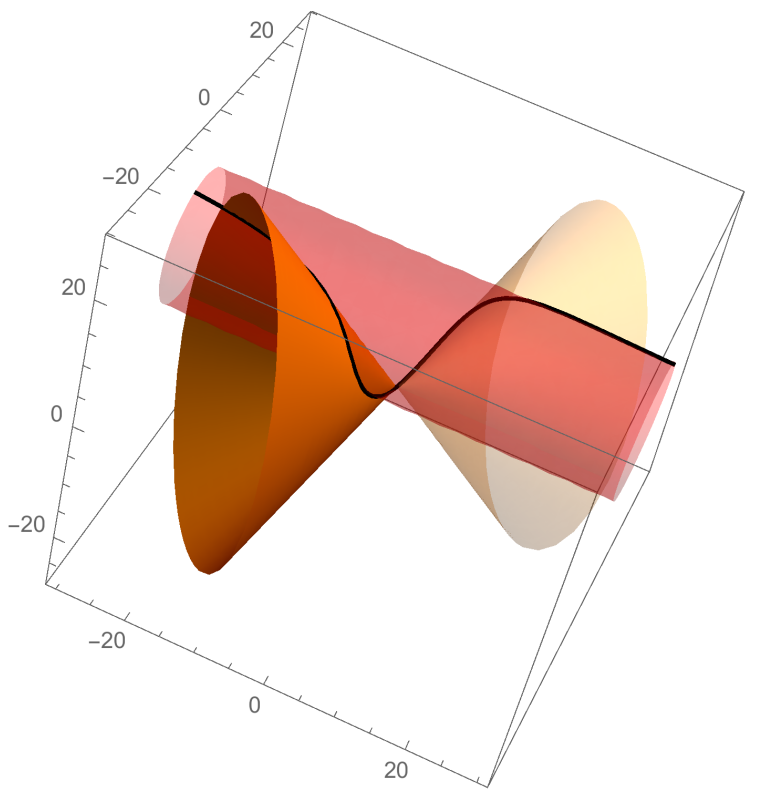

Fig. 1. Horopter curve at the intersection of a circular cylinder and an elliptic cone

different from zero, then this equation reduces to $\Pi_{\frac{z}{z}} w_{2}=0$. This implies that $z=\mu w_{2}\left(\mu \in \mathbb{R}^{*}\right)$. This is the equation of a straight line passing through the point $C$ and one recovers the already established fact that the body pose is not uniformly observable when all source points are on a straight line containing the point $C$. If $w_{2}=[0,0,0]^{\top}$ and $w_{1}$ is different from zero, then the equation reduces to $z \times w_{1}=0$ so that $z=\mu w_{1}$ $\left(\mu \in \mathbb{R}^{*}\right)$. This is also the equation of a straight line passing through the point $C$ and the same conclusion follows. A more interesting case is when neither $w_{1}$ nor $w_{2}$ are equal to zero. We show in the Appendix that in this case (20) is the equation of a family of horopter curves well known in photogrammetry [15], [16]. We further show that every horopter i) lies on a cylinder, the so-called "dangerous cylinder of space resection", ii) lies also on an elliptic cone whose apex, the point with zero coordinates, is on the curve. The horopter curve is thus the intersection of these two surfaces (see Figure 1) and it passes through the cone's apex, the point $C$ in the present case. This property does not seem to have been pointed out previously. Due to the specific role and location of the cone's apex on the curve we will be refer to it as the horopter's origin. One can show that the horopter is uniquely determined by four given reference points. Therefore, if four source points are located on a horopter curve (which is then uniquely defined), and if $C$ is the origin of the curve, then the body pose is not uniformly observable. Moreover, for a specific value of one of the horopter's parameters, the curve degenerates into a circle, perpendicular to the cylinder's axis, complemented with a straight line, parallel to the cylinder's axis, that intersects the circle at the point opposite to the horopter's origin w.r.t. the circle's centre. An ever more degenerate case is when the circle shrinks to a point on the straight line, in which case the horopter is a straight line that passes through the origin. One deduces (this can also be verified directly from (20)) that the body pose is also not uniformly observable when all source points lie on a degenerate horopter. Such is the case when all source points are aligned without the point $C$ being necessarily aligned with the source points (a case addressed previously), or when they are located on a circle containing also the point $C$, or when the source points are distributed on the circle and the straight line of a degenerate horopter whose origin is the point $C$. In all other cases the body pose is uniformly observable. The same results holds for more than four source points.

\section{Inertial velocity measurement}

Alike the mobile velocity measurement case one can derive different Riccati observers by considering various parametrizations of the body pose and, in particular, by considering again the four parametrizations $(\lambda, p),(\bar{\lambda}, p)$, $(\lambda, \bar{p})$ and $(\bar{\lambda}, \bar{p})$. We will limit the exposition of this case to the sole parametrization $(\lambda, p)$.

1) Observer based on the parametrization $(\lambda, p)$ : In this case $\dot{p}=v(t)$ with $v(t)$ being measured. Setting $x_{1}=2 \lambda$, $x_{2}=p$ and using the second equation of (13) one obtains the system equation (3) with

$$
A=0_{6}, u_{1}=\hat{R} \tilde{\omega}, u_{2}=v
$$

Concerning the output function $y$, one has for $i=1, \ldots, l$

$$
\begin{aligned}
\left|p-z_{i}\right| d_{i}= & R^{\top}\left(p-z_{i}\right) \\
= & \hat{R}^{\top} \tilde{R}^{\top}\left(p-z_{i}\right) \\
= & \hat{R}^{\top}\left(p-z_{i}\right)+\hat{R}^{\top}\left(\tilde{R}^{\top}-I_{3}\right)\left(p-z_{i}\right) \\
= & \hat{R}^{\top}\left(p-z_{i}\right)-\hat{R}^{\top} S(2 \lambda)\left(p-z_{i}\right) \\
& +O\left(\left|p-z_{i}\right||\lambda|^{2}\right) \\
= & \hat{R}^{\top}\left(p-z_{i}\right)+\hat{R}^{\top} S\left(\hat{p}-z_{i}\right)(2 \lambda) \\
& +O\left(\left|p-z_{i}\right||\lambda|^{2}\right)+O(|\lambda||\tilde{p}|)
\end{aligned}
$$

so that, by setting $y_{i}(\lambda, p, t):=\Pi_{d_{i}(t)} \hat{R}^{\top} z_{i}$, using the identity $\Pi_{d_{i}} d_{i}=0$ and the assumed boundedness of $\left|p(t)-z_{i}\right|$, one obtains

$$
\begin{aligned}
y_{i}(\lambda, p, t)= & \Pi_{d_{i}(t)} \hat{R}^{\top} S\left(\hat{p}-z_{i}\right)(2 \lambda)+\Pi_{d_{i}(t)} \hat{R}^{\top} p \\
& +O\left(|\lambda|^{2}\right)+O(|\lambda||\tilde{p}|)
\end{aligned}
$$

Therefore, by defining $y:=\left(y_{1}^{\top}, \ldots, y_{l}^{\top}\right)^{\top}$, one obtains the output equation (4) with

$$
\begin{gathered}
C_{1}\left(x_{1}, \hat{x}_{2}, t\right)=\left[\begin{array}{c}
\Pi_{d_{1}(t)} \hat{R}^{\top} S\left(\hat{x}_{2}-z_{1}\right) \\
\vdots \\
\Pi_{d_{l}(t)} \hat{R}^{\top} S\left(\hat{x}_{2}-z_{l}\right)
\end{array}\right] \\
C_{2}\left(x_{1}, t\right)=\left[\begin{array}{c}
\Pi_{d_{1}(t)} \hat{R}^{\top} \\
\vdots \\
\Pi_{d_{l}(t)} \hat{R}^{\top}
\end{array}\right]
\end{gathered}
$$

The corresponding Riccati observer giving the expressions of $\hat{\omega}$ and $\dot{\hat{p}}$ (that are used to compute $\hat{R}(t)$ and $\hat{p}(t)$ via numerical integration) is then given by (5)-(7) and (9). 
2) Observability issues: From the expressions of $A$ and $C$ one deduces that $A^{\star}=A=0_{6}$, so that $\Phi^{\star}(s, t)=I_{6}$, and

$$
C^{\star}(t)=\left[\begin{array}{cc}
\Pi_{d_{1}(t)} R^{\top}(t) S\left(p(t)-z_{1}\right) & \Pi_{d_{1}(t)} R^{\top}(t) \\
\vdots & \vdots \\
\Pi_{d_{l}(t)} R^{\top}(t) S\left(p(t)-z_{l}\right) & \Pi_{d_{l}(t)} R^{\top}(t)
\end{array}\right]
$$

Therefore, in this case

$$
\begin{aligned}
& \Delta(s, t):=\Phi^{\star \top}(s, t) C^{\star \top}(s) C^{\star}(s) \Phi^{\star}(s, t)= \\
& \sum_{i=1}^{l}\left[\begin{array}{c}
S^{\top}\left(p(s)-z_{i}\right) \\
I_{3}
\end{array}\right] \Pi_{d_{i}^{\mathcal{F}}(s)}\left[S\left(p(s)-z_{i}\right) I_{3}\right]
\end{aligned}
$$

and the observability Grammian is never positive if there exists $w \in \mathbb{R}^{6}-\{0\}$ such that

$$
\Pi_{d_{i}^{\mathcal{F}}(t)}\left[S\left(p(t)-z_{i}\right) \quad I_{3}\right] w=0, \forall i \in\{1, \ldots, l\}, \forall t \geq 0
$$

This relation is to be compared with the condition of nonuniform observability (16) associated with mobile velocity measurements. When the body position is constant, i.e. when $v(t) \equiv 0$, these two relations are equivalent. To verify this one can use the change of coordinates $w=\left[\begin{array}{c}\bar{w}_{1} \\ \bar{w}_{2}-p \times \bar{w}_{1}\end{array}\right]$. Then (22) is the same as (16) with $w$ replaced by $\bar{w}$. This equivalence was expected since, in the zero velocity case, the observers derived by assuming either mobile or inertial velocity measurements coincide. However, there is an important difference in the case of body motion. Indeed, we have previously established that, in the mobile velocity measurement case, at least three source points are required to grant uniform observability of the body pose, whether the point $C$ moves or is motionless. We show next that, when the inertial translational velocity of the body is measured and a single source point is used, the body pose is not uniformly observable only for very specific motions of the point $C$. In other words, body motion is better exploited for pose estimation in the sense that this estimation can be effectively performed with less information about the environment. Since the motionless case has been treated previously, only persistent $C$ translational motion is now considered.

\section{Single source point}

To simplify, one may arbitrarily assume that the source point $P$ coincides with the origin of the inertial frame, i.e. $z=[0,0,0]^{\top}$. Defining $d(t):=p(t) /|p(t)|$, the non-uniform observability condition (22) is then

$$
\Pi_{d(t)}\left[S(p(t)) \quad I_{3}\right] w=0, \forall t \geq 0
$$

or, equivalently, using the fact that $\Pi_{d(t)} S(p(t))=S(p(t))$

$$
p(t) \times w_{1}+\Pi_{d(t)} w_{2}=0, \quad \forall t \geq 0
$$

with $w=\left(w_{1}^{\top}, w_{2}^{\top}\right)^{\top}$. This equation indicates that $p(t)$ must satisfy for all $t$ the horopter equation (20) introduced previously, with the source point being now the origin of the horopter curve. The next proposition follows immediately:

Proposition 4.1: The body pose is not uniformly observable in the following situations

1) $C$ moves along a horopter curve whose origin is the source point,
2) $C$ moves along a straight line, or on a circle passing through the source point (the degenerate horopter's case).

Two and more source points

Since a non-degenerate horopter curve has a unique origin (that cannot coincide with two different source points), $C$ motion along such a curve does not make the body pose non-observable uniformly. $C$ motion on a circle passing through two source points does not forbid uniform stability either. Therefore non-observability occurs only when all source points and $C$ are aligned. One can then show that, independently of the location of the source points, a sufficient condition for uniform observability is the existence of $\delta>0$ and $\epsilon>0$ such that $\forall t \geq 0: \int_{t}^{t+\delta} v(s) v(s)^{\top} d s>\epsilon$. Note that this latter condition is not sufficient in the single source point case because the inequality can be satisfied by motions along a non-degenerate horopter.

\section{Simulations}

\section{A. Three source points and mobile velocity measurements}

This simulation illustrates that motion of $C$ on the danger cylinder, by contrast with the motionless case, allows for uniform observability and effective pose estimation. All distances are expressed in meters, and we call the inertial plane $\left\{O ; e_{1}, e_{2}\right\}$ the horizontal plane. The considered three source points are on this plane with coordinates respectively equal to $(0,0,0),(5,0,0)$ and $(2.5,2.5,0)$. The circle passing through them is centred at $(2.5,0,0)$ and has a radius equal to 2.5. The point $C$ moves on a circle parallel to it, ten meters above it and with a $1 \mathrm{~m} / \mathrm{s}$ translational velocity. Its coordinates are $p(t)=(2.5+2.5 \cos (0.4 t), 2.5 \sin (0.4 t), 10)^{\top}$ so that $v(t)=(-\sin (0.4 t), \cos (0.4 t), 0)$. The body's angular velocity is $\omega=(0.1 \sin (t), 0.4 \cos (2 t), 0.6 t)^{\top} \mathrm{rad} / \mathrm{s}$. For the Riccati pose observer we choose the parametrization $(\bar{\lambda}, \bar{p})$ and use, to simplify, a diagonal matrix $Q=\operatorname{diag}\left\{q_{1} I_{3}, \ldots, q_{l} I_{3}\right\}$. The observer equations are then

$$
\begin{aligned}
{\left[\begin{array}{c}
\hat{\omega} \\
\dot{\hat{p}}
\end{array}\right]=} & {\left[\begin{array}{c}
\omega \\
-S(\omega) \hat{\bar{p}}+\bar{v}
\end{array}\right] } \\
& -k P\left[\begin{array}{c}
\sum_{i=1}^{l} q_{i} S\left(\hat{R}^{\top} z_{i}\right) \Pi_{d_{i}}\left(\hat{\bar{p}}-\hat{R}^{\top} z_{i}\right) \\
\sum_{i=1}^{l} q_{i} \Pi_{d_{i}}\left(\hat{\bar{p}}-\hat{R}^{\top} z_{i}\right)
\end{array}\right] \\
\dot{\hat{R}}= & \hat{R} S(\hat{\omega}) \\
\dot{P}= & A P+P A^{\top}-P C^{\top} Q C P+V
\end{aligned}
$$

with $k(t) \geq 0.5$ and

$$
\begin{aligned}
& A=\left[\begin{array}{cc}
-S(\omega) & 0_{3 \times 3} \\
0_{3 \times 3} & -S(\omega)
\end{array}\right] \\
& C=\left[\begin{array}{cc}
-\Pi_{d_{1}} S\left(\hat{R}^{\top} z_{1}\right) & \Pi_{d_{1}} \\
\vdots & \vdots \\
-\Pi_{d_{l}} S\left(\hat{R}^{\top} z_{l}\right) & \Pi_{d_{l}}
\end{array}\right]
\end{aligned}
$$

with $l=3$. For the reported simulation results we have taken $k=1$ (as for a Kalman filter), $q_{i}=10(i=1,2,3), V=$ $\operatorname{diag}\left\{0.1 I_{3}, I_{3}\right\}$ and $P(0)=\operatorname{diag}\left\{I_{3}, 100 I_{3}\right\}$. Initial state values are $p(0)=(2.5,0,10)^{\top}$ and $R(0)=I_{3}$, whereas initial 
estimates are $\hat{p}(0)=(-2,4,3)^{\top}$ and $\bar{\Lambda}(0)=\left(\frac{\sqrt{2}}{2}, \frac{\sqrt{2}}{2}, 0,0\right)^{\top}$. This corresponds to an initial orientation angle error of 90 degrees.

Figures 2 (a) and (b) show the exponential convergence of the estimations errors to zero when the measurements of $\bar{v}(t)$ $\omega(t)$ and of the source points bearings are free of noise.

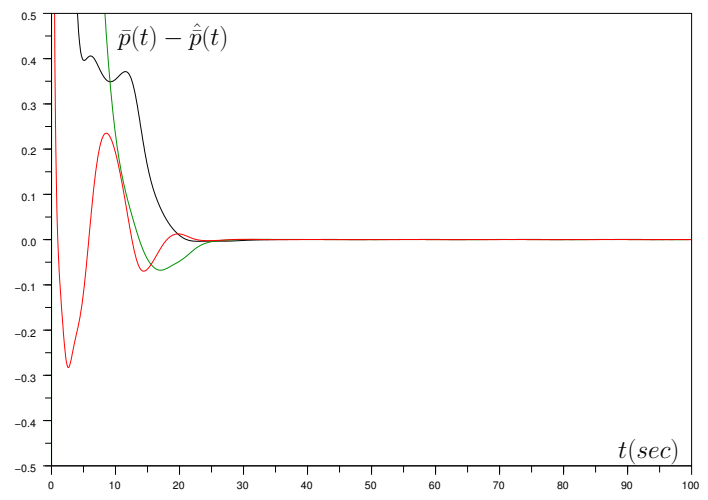

(a)

Fig. 2. Position error with noise-free measurements

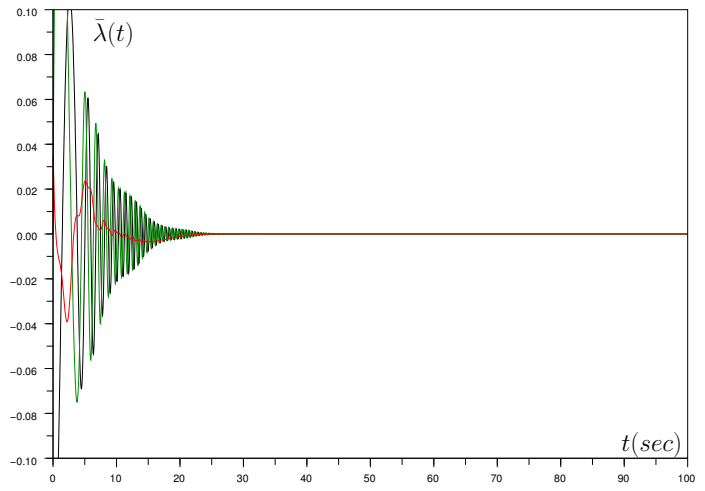

(b)

Fig. 2. Orientation error with noise-free measurements

Figures 3 (a) and (b) show the time-evolution of the same estimation errors and illustrate the performance of the same observer in the case of noisy measurements. These results have been obtained by replacing $\bar{v}(t)=R^{\top}(t) v(t)$ and $\omega(t)$ respectively by $R^{\top}(t) v(t)+b_{v}(t)$ and $\omega(t)+b_{\omega}(t)$ with $b_{v}$ and $b_{\omega}$ denoting vectors of uncorrelated zero-mean Gaussian noises with standard deviations equal to 0.1 (for the components of $b_{v}$ ) and 0.01 (for the components of $b_{\omega}$ ). As for the bearing measurements we have simulated noisy position measurements of the source points seen in the images of a calibrated camera by replacing $d_{i}(i=1,2,3)$ by

$$
\begin{aligned}
& d_{i}^{\text {mes }}=\frac{\operatorname{sign}\left(d_{i, 3}\right)}{\text { denom }}\left(d_{i, 1} / d_{i, 3}+n_{i, 1}, d_{i, 2} / d_{i, 3}+n_{i, 2}, 1\right)^{\top} \\
& \text { denom }=\sqrt{\left(d_{i, 1} / d_{i, 3}+n_{i, 1}\right)^{2}+\left(d_{i, 2} / d_{i, 3}+n_{i, 2}\right)^{2}+1}
\end{aligned}
$$

with $n_{i, 1}$ and $n_{i, 2}$ denoting uncorrelated zero-mean uniformly distributed noises with maximum deviation equal to 0.005 . For a CCD camera with an aperture of 90 degrees and producing images with $(1000 \times 1000)$ pixels this corresponds approximately to a maximum localisation error of 2.5 pixels, or $50 \mathrm{~cm}$ in any direction at a distance of 10 meters. By comparison, the ultimate maximum estimation error of about $10 \mathrm{~cm}$ observed in Figure 3 (a) illustrates the filtering property of the observer.

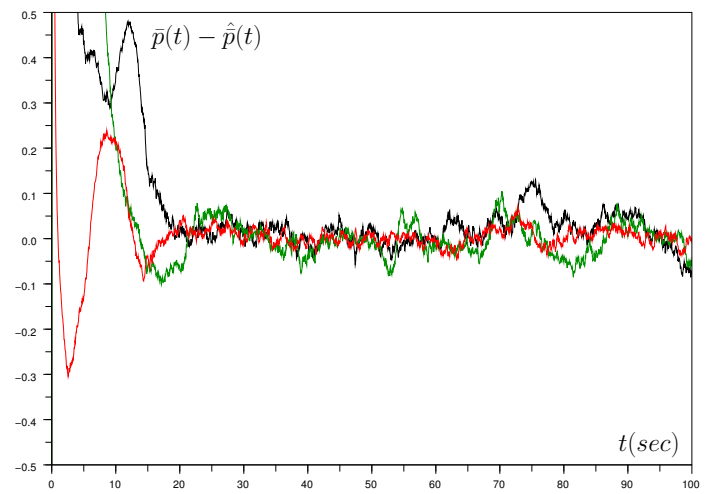

(a)

Fig. 3. Position error with noisy measurements

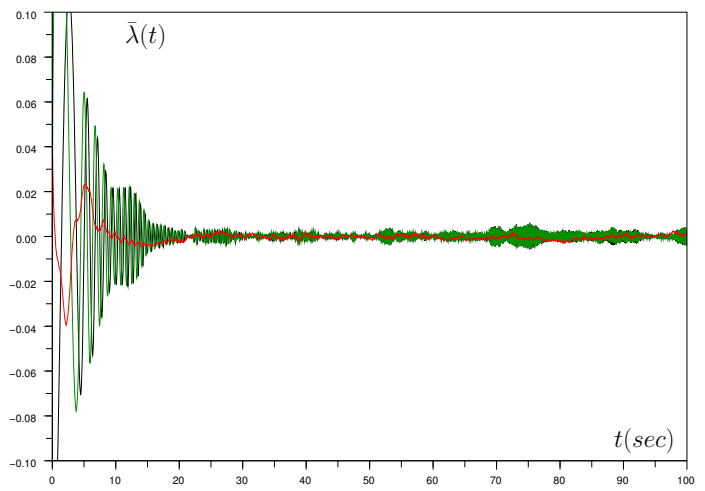

(b)

Fig. 3. Orientation error with noisy measurements

\section{B. One source point and inertial velocity measurements}

These simulations illustrate the possibility of estimating the body pose with bearing measurements of a single source point when the point $C$ moves and its velocity is measured in the inertial frame. For the Riccati pose observer we choose the parametrization $(\lambda, p)$ and use again, to simplify, a diagonal matrix $Q=\operatorname{diag}\left\{q_{1} I_{3}, \ldots, q_{l} I 3\right\}$. In the case of $l$ source points, the observer equations are

$$
\begin{aligned}
{\left[\begin{array}{c}
\hat{\omega} \\
\dot{\hat{p}}
\end{array}\right] } & =\left[\begin{array}{c}
\omega \\
v
\end{array}\right] \\
& -k\left[\begin{array}{cc}
\hat{R}^{\top} & 0_{3} \\
0_{3} & I_{3}
\end{array}\right] P\left[\begin{array}{c}
\sum_{i=1}^{l} q_{i} \hat{R} S^{\top}\left(\xi_{i}\right) \Pi_{d_{i}} \xi_{i} \\
\sum_{i=1}^{l} q_{i} \hat{R} \Pi_{d_{i}} \xi_{i}
\end{array}\right] \\
\dot{\hat{R}} & =\hat{R} S(\hat{\omega}) \\
\dot{P} & =-P C^{\top} Q C P+V
\end{aligned}
$$


with $\xi_{i}:=\hat{R}^{\top}\left(\hat{p}-z_{i}\right), k(t) \geq 0.5$, and

$$
C=\left[\begin{array}{cc}
\Pi_{d_{1}} S\left(\xi_{1}\right) \hat{R}^{\top} & \Pi_{d_{1}} \hat{R}^{\top} \\
\vdots & \vdots \\
\Pi_{d_{l}} S\left(\xi_{l}\right) \hat{R}^{\top} & \Pi_{d_{l}} \hat{R}^{\top}
\end{array}\right]
$$

In the present case, $l=1$. The single source point used for pose estimation is the first of the three source points used in the previous simulations, i.e. $z_{1}=[0,0,0]^{\top}$. The point $C$ again moves on a cylinder passing through the source point, along a horizontal circular trajectory this time located 5 meters above the source point, and with a translational velocity 2.5 times larger than in the previous simulations, i.e. $p(t)=[2.5+2.5 \cos (t), 2.5 \sin (t), 5]^{\top}$. The reason for these modifications (smaller distance to the source point, larger velocity) is to amplify the excitation properties associated with the motion of $C$ in terms of observability, and subsequently increase the rate of convergence of the estimation errors to zero [1]. Initial pose estimates are $\hat{p}(0)=[3,2,7]^{\top}$ and $\Lambda(0)=$ $[\sqrt{3} / 2,0.5,0,0]^{\top}$. This corresponds to an initial rotation angle error of 60 degrees. The initial value of the Riccati matrix is $P(0)=\operatorname{diag}\left\{I_{3}, 10 I_{3}\right\}$. The other parameters entering the Riccati equation $(k, V$ and $Q)$ and the measurement noises are the same as in the previous simulations.

Figures 4 (a) and (b) show the exponential convergence of the estimations errors to zero when the measurements of $v(t)$, $\omega(t)$ and of the source point bearing are free of noise.

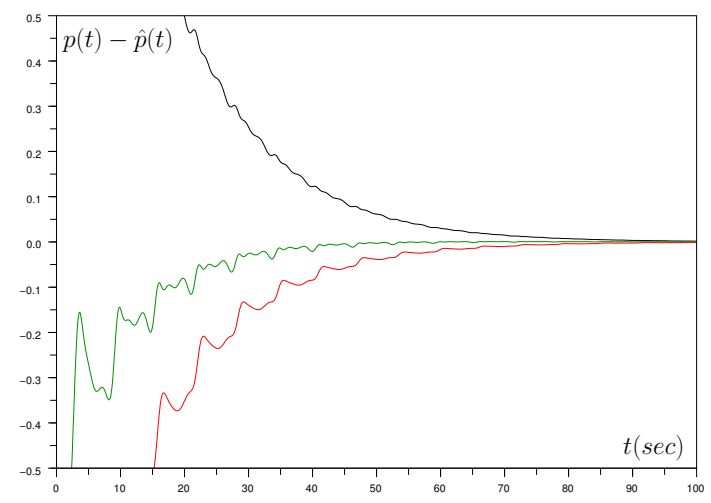

(a)

Fig. 4. Position error with noise-free measurements

Figures 5 (a) and (b) show the time-evolution of the same estimation errors and illustrate the performance of the observer in the case of noisy measurements.

\section{STEREO VISION VS. MONOCULAR VISION}

A stereo vision system consists in the rigid pairing of two (usually identical) cameras whose optic axes are parallel and orthogonal to the line joining the cameras' optic centres. Let the mid-point between the optic centres be the origin $C$ of the mobile frame rigidly linked to the cameras, and $a$ (resp. $-a$ ) the known vector of coordinates of one of the optic centres (resp. of the other optic centre) expressed in the mobile frame basis. Define also $p_{z_{i}}:=R^{\top}\left(p-z_{i}\right)$, i.e. the opposite of the vector of coordinates of the $i t h$ source point expressed in the

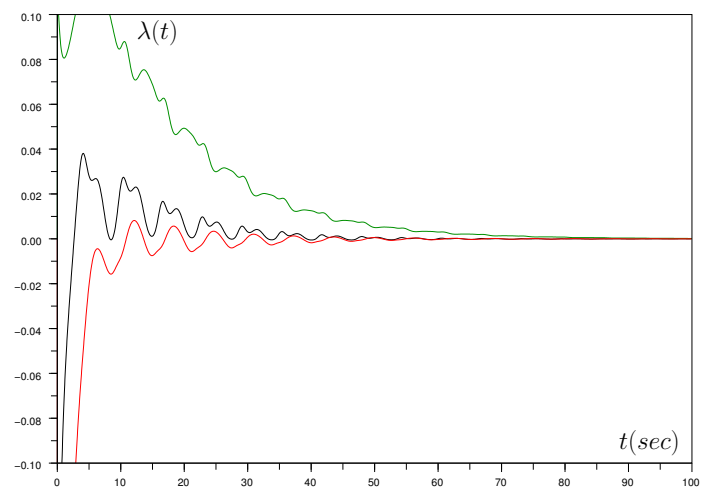

(b)

Fig. 4. Orientation error with noise-free measurements

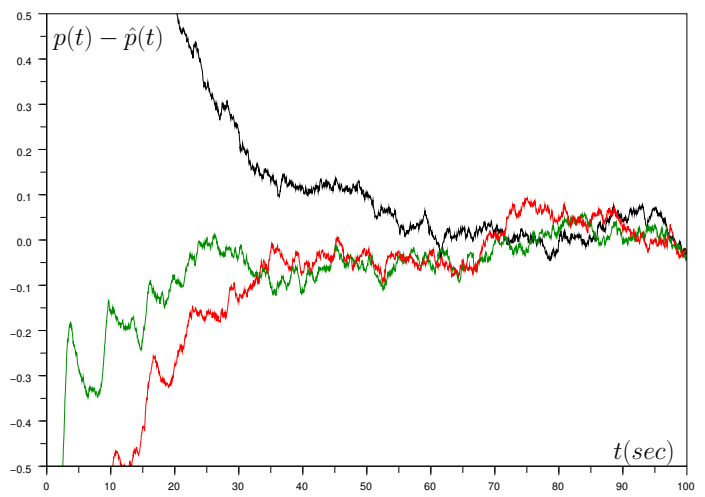

(a)

Fig. 5. Position error with noisy measurements

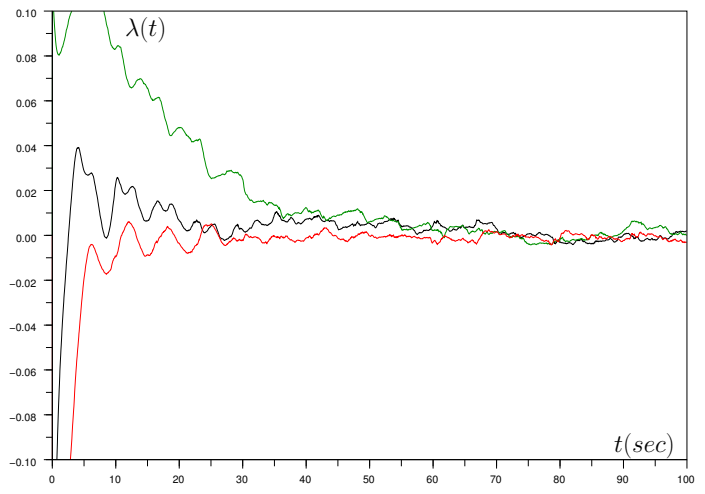

(b)

Fig. 5. Orientation error with noisy measurements

mobile frame basis, and denote the bearing of the ith source point measured from the image of the first (resp. second) camera by $d_{i, 1}\left(\right.$ resp. $\left.d_{i, 2}\right)$, i.e. $d_{i, 1}=\frac{p_{z_{i}}+a}{\left|p_{z_{i}}+a\right|}$ and $d_{i, 2}=\frac{p_{z_{i}}-a}{\left|p_{z_{i}}-a\right|}$. Then $d_{i, 1} \times d_{i, 2}=\frac{2 a \times p_{z_{i}}}{\left|p_{z_{i}}+a\right|\left|p_{z_{i}}-a\right|}, a \times d_{i, 1}=\frac{a \times p_{z_{i}}}{\left|p_{z_{i}}+a\right|}$ and $a \times d_{i, 2}=\frac{a \times p_{z_{i}}}{\left|p_{z_{i}}-a\right|}$. Therefore $\left|p_{z_{i}}-a\right|=2 \frac{\left|a \times d_{i, 1}\right|}{\left|d_{i, 1} \times d_{i, 2}\right|}$ and $\left|p_{z_{i}}+a\right|=2 \frac{\left|a \times d_{i, 2}\right|}{\left|d_{i, 1} \times d_{i, 2}\right|}$. Since $p_{z_{i}}=d_{i, 1}\left|p_{z_{i}}+a\right|-a$ (resp. $\left.p_{z_{i}}=d_{i, 2}\left|p_{z_{i}}-a\right|+a\right)$ one deduces that $p_{z_{i}}=2 \frac{d_{i, 1}\left|a \times d_{i, 2}\right|}{\left|d_{i, 1} \times d_{i, 2}\right|}-a$ 
(resp. $\left.p_{z_{i}}=2 \frac{d_{i, 2}\left|a \times d_{i, 2}\right|}{\left|d_{i, 1} \times d_{i, 2}\right|}+a\right)$ and thus also

$$
p_{z_{i}}=\frac{d_{i, 1}\left|a \times d_{i, 2}\right|+d_{i, 2}\left|a \times d_{i, 2}\right|}{\left|d_{i, 1} \times d_{i, 2}\right|}
$$

This latter relation establishes the known fact that a stereo vision system allows for the measurement of the observed source point position expressed in the cameras' frame, whereas a monocular vision system only allows for the source point bearing measurement. From there Riccati observers can be derived in the same way as for a single camera either by using source point(s) bearings measured in the images of the two cameras, without computing the $p_{z_{i}}(i=1, \ldots, l)$ explicitly, or by using the $p_{z_{i}}$ given by (26). In both cases one can verify that the non-uniform observability conditions (16) and (22) become

$$
\left[S^{\top}\left(z_{i}\right) \quad I_{3}\right] w=0, \forall i \in\{1, \ldots, l\}, \forall t \geq 0
$$

for some $w \neq 0$, when the body translational velocity is measured in the mobile frame, and

$$
\left[S\left(p(t)-z_{i}\right) \quad I_{3}\right] w=0, \forall i \in\{1, \ldots, l\}, \forall t \geq 0
$$

for some $w \neq 0$, when this velocity is measured in the inertial frame. These conditions are thus simply obtained by replacing the projection operators $\Pi_{d_{i}^{\mathcal{F}}}$ by the identity matrix $I_{3}$.

The first of these conditions tells us that, when the velocity of $C$ is measured in the mobile frame, the body pose is not observable in the case of one and two source points, and that it is uniformly observable in the case of three or more nonaligned source points. These results are thus essentially the same as when using a monocular vision system, except for the non-existence of a danger cylinder in the three source points case and of specific singular body motions that do not grant uniform observability when three or more source points are used. However, a more significant difference, when using three or more non-aligned source points whose positions are known in advance, results from the possibility of estimating the body attitude independently of the body position in this case. Indeed, almost global convergence to zero of the pose estimation error can then be proved despite the approximations made when deriving the equations of the Riccati observer (see, for instance, [17] where the identity matrix is implicitly used as a solution to the CRE associated with the attitude observer). A complementary interest of using a stereo camera is that the measurement/estimation of three (or more) source points positions expressed in the camera's frame can be performed during an initialization phase when the camera is kept motionless. By interpreting the camera's initial frame as the inertial frame w.r.t which the camera's pose is subsequently estimated, one is brought back to the previously evoked case where the position of at least three source points positions are known in advance, so that almost global convergence to zero of the pose estimation error, independently of the frame w.r.t. which the camera's translational velocity is measured, can be achieved in this case. From this result one readily infers that almost global convergence and exponential stability of stereo vision-based EKF-SLAM [18] algorithms, consisting of online body pose estimation complemented with online estimation of extra landmarks positions, can also been proved provided that the source points used for the initialization phase are always seen by the camera.

The second condition tells us that, in the case of persistent translational body motion, when the velocity is measured in the inertial frame and when a single source is used, the body pose is not uniformly observable when $C$ moves along a straight line, but also that the satisfaction of $\forall t \geq$ $0: \int_{t}^{t+\delta} v(s) v(s)^{\top} d s>\epsilon I_{3}$ for some $\delta$ and $\epsilon$ positive is sufficient to ensure uniform observability of the body pose. Conditions for uniform observability are thus again only slightly weaker than when using a monocular vision system.

\section{CONCLUding REMARKS}

In this paper, original Riccati body pose observers using measured body velocities and source points bearing measurements in the body frame are derived and analysed. Rigorous local exponential stability of these EKF-like observers, under specified uniform observability conditions, is proved. Situations for which these conditions are not met are characterized in details in terms of the number and location of the source points, and also in terms of the body position and motion relatively to the source points. Differences resulting from measuring the body translational velocity in an inertial frame rather than in the body frame are pointed out. Concerning this latter issue, the present study points out the importance of measuring the body translational motion in an inertial frame, especially when bearing measurements are obtained with a monocular camera attached to the body. Indeed, when this velocity is measured in the mobile frame, body motion only marginally modifies the observability conditions under which pose estimation can be performed efficiently. Basically, at least three source points whose positions are measured in the inertial frame are necessary in this case. By contrast, a single source point is generically sufficient when the body keeps moving and its velocity is measured in an inertial frame. Moreover, no measurement of the source point position is needed when the source point coincides with the origin of the chosen inertial frame w.r.t. which the body position is estimated. This latter fact enlightens the kinship between the localization problem in monocular vision-based SLAM [19] and the pose estimation problem addressed in the present paper: unless three landmarks positions are known initially, the localization problem is wellposed in terms of "consistence" (i.e. robust convergence of the pose estimation errors to zero) only when the body velocity is measured w.r.t. the chosen inertial frame. This is coherent with the fact that pose estimation w.r.t. an inertial frame is possible only when measurements made w.r.t. the inertial frame are available (the body velocity, in the present case). Using a stereoscopic camera alleviates this constraint by implementing an initial phase during which the camera is motionless and three (or more) source points positions are estimated in the camera's frame. This frame then becomes the inertial frame w.r.t. which the pose estimation is subsequently carried out. Connections with the SLAM problem will be further discussed in forthcoming studies. 


\section{APPENDIX}

\section{A. Horopter's equations}

We derive the horopter's equations from (20) when $w_{1}$ and $w_{2}$ are different from zero, and we show that the horopter may also be defined as the intersection of a circular cylinder with a cone asymptotically tangent to the cylinder.

Let $e_{i}(i=1,2,3)$ denote the canonical basis of $\mathbb{R}^{3}$, i.e. $e_{1}=(1,0,0)^{\top}, e_{2}=(0,1,0)^{\top}$ and $e_{3}=(0,0,1)^{\top}$. Since $w_{1}$ and $w_{2}$ are different from zero, one can arbitrarily set $w_{1}=e_{1}$ and $w_{2}=-\left(k e_{1}+a e_{2}\right)$, with $a \in \mathbb{R}$ and $k \in \mathbb{R}$ being then the curve's parameters. Equation (20) then becomes

$$
z \times e_{1}-\Pi_{\frac{z}{|z|}}\left(k e_{1}+a_{2}\right)=0
$$

or, equivalently

$$
|z|^{2}\left(z \times e_{1}\right)-\left(|z|^{2} I_{3}-z z^{\top}\right)\left(\left(k e_{1}+a_{2}\right)=0\right.
$$

Let $z_{i}(i=1,2,3)$ denote the coordinates of the vector $z$. Using these coordinates in the previous equality yields

$$
\begin{aligned}
& -k|z|^{2}+z_{1}\left(k z_{1}+a z_{2}\right)=0 \\
& |z|^{2}\left(z_{3}-a\right)+z_{2}\left(k z_{1}+a z_{2}\right)=0 \\
& -|z|^{2} z_{2}+z_{3}\left(k z_{1}+a z_{2}\right)=0
\end{aligned}
$$

therefore

$$
\frac{k z_{1}+a z_{2}}{|z|^{2}}=\frac{k}{z_{1}}=-\frac{z_{3}-a}{z_{2}}=\frac{z_{2}}{z_{3}}
$$

so that, in view of the last equalities, $z_{2}^{2}=z_{3}\left(a-z_{3}\right)$ and $z_{1}=k z_{3} / z_{2}$. Assume for the time being that $a>0$, then the positivity of $z_{2}^{2}$ implies that $z_{3} \in[0, a](\geq 0)$ and that $z_{2}= \pm \sqrt{z_{3}\left(a-z_{3}\right)}$. Therefore $z_{1}= \pm k \sqrt{z_{3}} / \sqrt{a-z_{3}}$. It just remains to verify that these equalities are compatible with the first equality of (29), i.e. $-k|z|^{2}+z_{1}\left(k z_{1}+a z_{2}\right)=0$ or, equivalently, $k\left(z_{2}^{2}+z_{3}^{2}\right)=a z_{1} z_{2}$. In view of the expression of $z_{2}$ in terms of $z_{3}$ one has $k\left(z_{2}^{2}+z_{3}^{2}\right)=a k z_{3}$ and, using the expression of $z_{1}$ and $z_{2}$ in terms of $z_{3}$ one has $a z_{1} z_{2}=a k z_{3}$. The expected equality thus holds true. The equations of the two curves obtained so far are

$$
z_{1}=k \sqrt{z_{3}} / \sqrt{a-z_{3}}, z_{2}=\sqrt{z_{3}\left(a-z_{3}\right)}, z_{3} \in[0, a)
$$

and

$$
z_{1}=-k \sqrt{z_{3}} / \sqrt{a-z_{3}}, \quad z_{2}=-\sqrt{z_{3}\left(a-z_{3}\right)}, z_{3} \in[0, a)
$$

The first set of equations are the common equations of the horopter, and the second set provides the same geometrical curve rotated by an angle $\pi$. With $a<0$ one also verifies that the same curves are again obtained modulo a symmetry w.r.t. the origin. Therefore all solutions to (20) yield the same geometrical horopter curve whose generic equations are given by $(30)$.

The equality $z_{2}^{2}+z_{3}^{2}-a z_{3}=0$ is satisfied by every point on the horopter. This is also the equation of a circular cylinder of diameter equal to $a$ and whose axis direction is given by $e_{1}$. Therefore the horopter lies on the surface of this cylinder. Another equality obtained previously is $k\left(z_{2}^{2}+z_{3}^{2}\right)-a z_{1} z_{2}=$ 0 . This is the equation of an elliptic cone whose apex is the horopter's origin, whose central axis direction is given by the vector $\left[a, \sqrt{a^{2}+k^{2}}-k, 0\right]^{\top}$ and whose geometric characteristic numbers are $d=\left(\sqrt{a^{2}+k^{2}}-k\right) /(2 k), e=$ $\left(\sqrt{a^{2}+k^{2}}-k\right) /\left(\sqrt{a^{2}+k^{2}}+k\right)$, and $f=1$ (see figure 6). Therefore, the horopter curve lies also on the surface of this

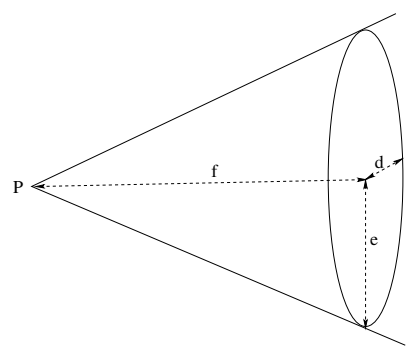

Fig. 6. Cone characteristic numbers

cone. The horopter curve is thus the intersection of a circular cylinder and an elliptic cone. Moreover, when $z_{3}$ tends to $a$, the coordinate $z_{1}$ tends to infinity. The horopter curve is thus unbounded and this implies that the cone is asymptotically tangent to the cylinder.

When the parameter $k$ tends to zero the horopter degenerates into a circle of diameter $a$ and a straight line perpendicular to the circle and intersecting it at the point opposite to the horopter's origin w.r.t. the circle's centre. When $a$ tends to zero the horopter further degenerates into a straight line containing the origin.

\section{B. Extension to the estimation of velocity biases}

Velocity measurements are often corrupted by biases that are constant or slowly varying. In this case it is useful to complement the observer with an estimation of these biases. Note that these biases may also be interpreted as unknown constant body velocities. This latter remark is of practical importance since it alleviates the necessity of measuring body velocities when these velocities are constant or slowly varying, except of course in the case of a single source point and when the body translational velocity is constant in the inertial frame since the body pose is then not observable by application of Proposition 4.1. An extension of the observer (25) is proposed next and its performance, when using a single source point and noisy measurements, is illustrated by simulation. The other Riccati observers considered in the present paper can easily be modified in the same manner.

Let $\omega_{b}$ denote the bias on the body's angular velocity so that $\frac{d}{d t} R(t)=R(t)\left(\omega(t)+\omega_{b}\right)$, with $\omega(t)$ the measured part of the angular velocity. Let similarly $v_{b}$ denote a bias on the body's translational velocity so that $\dot{p}(t)=v(t)+v_{b}$, with $v(t)$ the measured part of the translational velocity. Via a straightforward extension of Theorem 3.1 involving different dimensions for $x_{1}$ and $x_{2}$ so as incorporate $v_{b}$ and $\omega_{b}$ into the system's state vector, setting $\frac{d}{d t} \hat{R}=\hat{R} S\left(\hat{\omega}+\hat{\omega}_{b}\right)$ and using the approximation

$$
\begin{aligned}
2 \dot{\lambda} & =\hat{R}\left(\tilde{\omega}+\tilde{\omega}_{b}\right) \lambda_{0}+0.5 S\left(\hat{R}\left(\tilde{\omega}+\tilde{\omega}_{b}\right)\right) 2 \lambda \\
& =\hat{R}\left(\tilde{\omega}+\tilde{\omega}_{b}\right)+O(|\tilde{\omega}||\lambda|)+O\left(\left|\tilde{\omega}_{b}\right||\lambda|\right)
\end{aligned}
$$


one obtains the following equations of an extension of the Riccati observer (25)

$$
\begin{aligned}
& {\left[\begin{array}{c}
\hat{\omega} \\
\dot{\hat{p}} \\
\dot{\hat{\omega}}_{b} \\
\dot{\hat{v}}_{b}
\end{array}\right]=\left[\begin{array}{c}
\omega \\
v+\hat{v}_{b} \\
0_{3 \times 1} \\
0_{3 \times 1}
\end{array}\right]} \\
& -k\left[\begin{array}{cc}
\hat{R}^{\top} & 0_{3 \times 9} \\
0_{9 \times 3} & I_{9}
\end{array}\right] P\left[\begin{array}{c}
\sum_{i=1}^{l} q_{i} \hat{R} S^{\top}\left(\xi_{i}\right) \Pi_{d_{i}} \xi_{i} \\
\sum_{i=1}^{l} q_{i} \hat{R} \Pi_{d_{i}} \xi_{i} \\
0_{6 \times 1}
\end{array}\right] \\
& \dot{P}=A P+A^{\top} P-P C^{\top} Q C P+V
\end{aligned}
$$

with $\xi_{i}:=\hat{R}^{\top}\left(\hat{p}-z_{i}\right), k(t) \geq 0.5$,

$$
A=\left[\begin{array}{c|cc}
0_{6} & \hat{R} & 0_{3} \\
& 0_{3} & I_{3} \\
\hline 0_{6} & \multicolumn{2}{|c}{0_{6}}
\end{array}\right]
$$

and

$$
C=\left[\begin{array}{cccc}
\Pi_{d_{1}} S\left(\xi_{1}\right) \hat{R}^{\top} & \Pi_{d_{1}} \hat{R}^{\top} & 0_{3} & 0_{3} \\
\vdots & \vdots & \vdots & \vdots \\
\Pi_{d_{l}} S\left(\xi_{l}\right) \hat{R}^{\top} & \Pi_{d_{l}} \hat{R}^{\top} & 0_{3} & 0_{3}
\end{array}\right]
$$

For the simulation results reported next the same source point, translational and angular body motions, and measurement noises as for the case of unbiased velocities are used. State initial conditions, complemented with zero bias estimates, are also the same. The biases on the translational and angular velocities are $v_{b}=[0.1,-0.05,0.2]^{\top}$ and $\omega_{b}=$ $[0.01,0.004,-0.02]^{\top}$ respectively. Figures (7) (a) and (b) show the time-evolution of the position and attitude estimation errors, whereas Figures (7) (c) and (d) show the time-evolution of the velocity biases estimation errors. The convergence of all estimation errors to small values illustrates the performance of the observer.

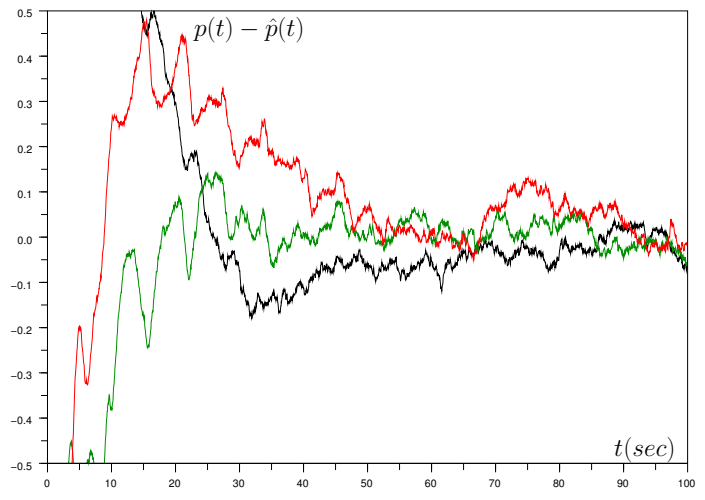

(a)

Fig. 7. Position error

\section{REFERENCES}

[1] T. Hamel and C. Samson, "Position estimation from direction or range measurements," Automatica, vol. 82, pp. 137-144, 2017.

[2] R. Haralick, C. Lee, K. Ottenberg, and M. Nölle, "Review and analysis of solutions of the three point perspective pose estimation problem," International Journal of Computer Vision, vol. 13, no. 3, pp. 331-356, 1994.

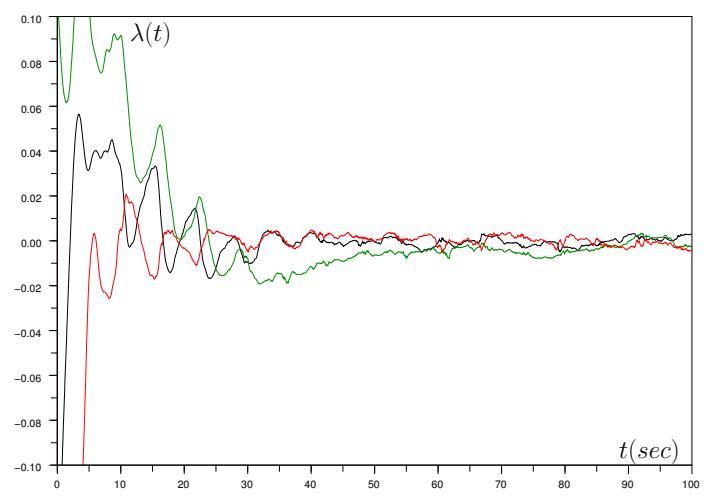

(b)

Fig. 7. Orientation error

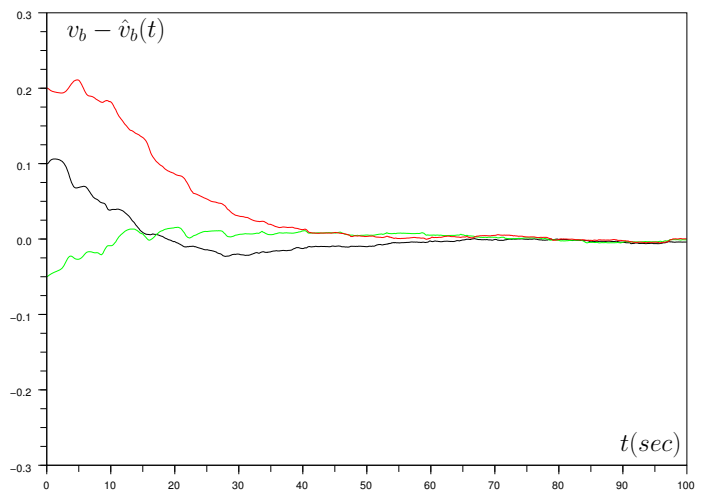

(c)

Fig. 7. Translational velocity bias error

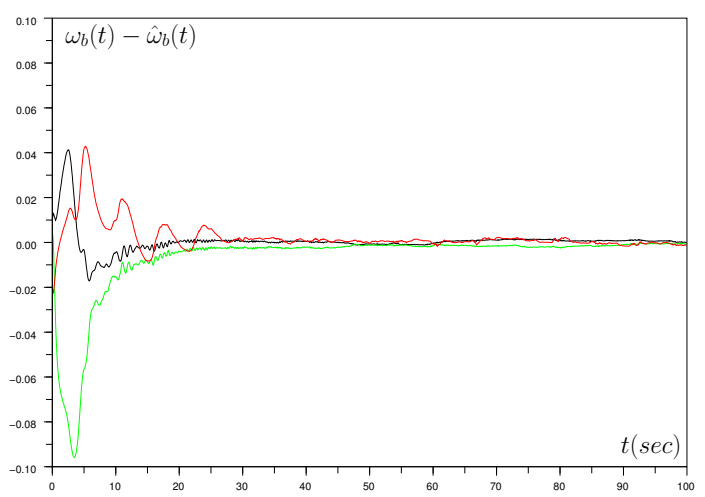

(d)

Fig. 7. Angular velocity bias error

[3] J. Grunert, "Das pothenotische problem in erweiterter gestalt nebst über seine anwendungen in der geodäsie," Grunerts Archiv für Mathematik und Physik, Band 1, pp. 238-248, 1841.

[4] M. Fischer and R. Bolles, "Random sample consensus: A paradigm for model fitting with applications to image analysis and automated cartography," Graphics and Image Processing, vol. 24, no. 6, pp. 381395, 1981.

[5] M. Dhome, M. Richetin, J. Lapreste, and . Rives, "The inverse perspective problem from a single view for polyhedra location," in IEEE Conference on Computer Vision and Pattern Recognition, 1988, pp. 6168

[6] L. Kneip, D. Scaramuzza, and R. Siegwart, "A novel parametrization 
of the perspective-three-point problem for a direct computation of absolute camera position and orientation," in Computer Vision and Pattern Recognition (CVPR), 2011 IEEE Conference on. IEEE, 2011, pp. 2969-2976.

[7] $\mathrm{Z}$. $\mathrm{Hu}$ and F. Wu, "A note on the number of solutions of the noncoplanar p4p problem," IEEE Trans. on Pattern Analysis and Machine Intelligence, vol. 24, no. 3, pp. 1-6, 2002.

[8] R. Haralick, H. Joo, C. Lee, X. Zhuang, V. Vaidya, and M. Kim, "Pose estimation from corresponding point data," IEEE transactions on Systems, Man and Cybernetics, vol. 19, no. 6, pp. 1426-1446, 1989.

[9] C.-P. Lu, G. Hager, and E. Mjolsness, "Fast and globally convergent pose estimation from video images," IEEE Trans. on Pattern Analysis and Machine Intelligence, vol. 22, no. 6, pp. 610-622, 2000.

[10] A. Aguiar and J. Hespanha, "Minimum-energy state estimation for systems with perspective outputs," IEEE Trans. on Automatic Control, vol. 51, no. 2, pp. 226-241, 2006.

[11] G. Baldwin, R. Mahony, and J. Trumpf, "A nonlinear observer for 6 DOF pose estimation from inertial and bearing measurements," in Proceedings of the IEEE International Conference on Robotics and Automation (ICRA), 2009, pp. 2237-2242.

[12] C.-T. Chen, Linear System Theory and Design, 2nd ed. CBS College Publishing, 1984.

[13] F. Markley, "Attitude error representations for kalman filtering," Journal of Guidance, Control, and Dynamics, vol. 26, no. 2, pp. 311-317, 2003.

[14] A. Loria and E. Panteley, "Uniform exponential stability of linear timevarying systems: revisited," Systems \& Control Letters, vol. 47, no. 1, pp. 13-24, 2002

[15] S. Finsterwalder, "Die geometrischen grundlagen der ptotogrammetrie," Jahresbericht Deutsche Mathem. Vereinigung, vol. 6, no. 2, pp. 1-41, 1899.

[16] B. Wrobel, "Calibration and orientation of cameras in computer vision," Springer Series in Information Sciences, vol. 34, pp. 7-62, 2001.

[17] M.-D. Hua, M. Zamani, J. Trumpf, R. Mahony, and T. Hamel, "Observer design on the special euclidean group se(3)," in 50th IEEE Conference on Decision and Control (CDC). IEEE, 2011, pp. 8169-8175.

[18] T. Lemaire, C. Berger, I. Jung, and S. Lacroix, "Vision-based slam Stereo and monocular approaches," Int. J. of Computer Vision, vol. 74 no. 3, pp. 343-364, 2007.

[19] A. Davison, I. Reid, N. Molton, and O. Stasse, "Monoslam: Real-time single camera slam," IEEE Trans. on Pattern Analysis and Machine Intelligence, vol. 29, no. 6, pp. 1-15, 2007.

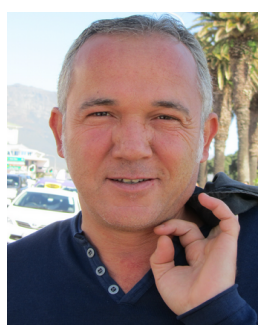

Tarek Hamel is Professor at the University of Nice Sophia Antipolis since 2003. He received his $\mathrm{Ph} . \mathrm{D}$. degree in Robotics from the University of Technology of Compiègne (UTC), France, in 1996. After two years as a research assistant at the UTC he joined the Centre d'Etudes de Mécanique d'Ile de France in 1997 as an associate professor. His research interests include nonlinear control theory, estimation and vision-based control with applications to Unmanned Aerial Vehicles. He is currently Associate Editor for IEEE Transactions on Robotics and was Associate Editor for Control Engineering Practice from 2010 to 2015

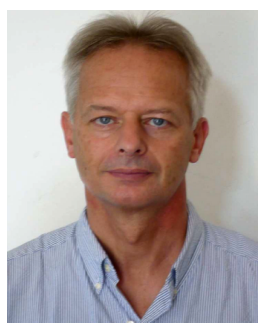

Claude Samson graduated from the Ecole Supérieure d'Electricité in 1977, and received his Docteur-Ingénieur and Docteur d'Etat degrees from the University of Rennes, in 1980 and 1983, respectively. He joined INRIA in 1981, where he is presently Directeur de Recherche. His research interests are in control theory and its applications to the control of mechanical systems. Dr. Samson is the coauthor, with M. Leborgne and B. Espiau, of the book Robot Control. The Task-Function Approach (Oxford University Press, 1991). 